\title{
Phenomenology of the hidden SU(2) vector dark matter model
}

\author{
Nabil Baouche, ${ }^{1,2, *}$ Amine Ahriche $\odot^{3,4, \dagger}$ Gaber Faisel $\oplus^{5, \$}$ and Salah Nasri $\oplus^{6,4, \S}$ \\ ${ }^{1}$ Faculty of Science and Technology, University of Jijel PB 98 Ouled Aissa, DZ-18000 Jijel, Algeria \\ ${ }^{2}$ Laboratoire de Physique des Particules et Physique Statistique, Ecole Normale Superieure, \\ BP 92 Vieux Kouba, DZ-16050 Algiers, Algeria \\ ${ }^{3}$ Department of Applied Physics and Astronomy, University of Sharjah, P.O. Box 27272 Sharjah, UAE \\ ${ }^{4}$ The Abdus Salam International Centre for Theoretical Physics, Strada Costiera 11, I-34014, Trieste, Italy \\ ${ }^{5}$ Department of Physics, Faculty of Arts and Sciences, Süleyman Demirel University, \\ Isparta, Turkey 32260 \\ ${ }^{6}$ Department of Physics, United Arab Emirates University, Al-Ain, UAE
}

(Received 5 June 2021; accepted 12 September 2021; published 14 October 2021)

\begin{abstract}
We investigate the phenomenology of an extension of the Standard Model (SM) by a non-Abelian gauge group $\mathrm{SU}(2)_{\mathrm{HS}}$ where all SM particles are singlets under this gauge group, and a new scalar representation $\phi$ that is singlet under $\mathrm{SM}$ gauge group and doublet under $\mathrm{SU}(2)_{\mathrm{HS}}$. In this model, the dark matter (DM) candidates are the three mass-degenerate dark photons $A_{i}(i=1,2,3)$ of $\mathrm{SU}(2)_{\mathrm{HS}}$, and the hidden sector interacts with the (SM) particles through the Higgs portal interactions. Consequently, there will be a new $C P$-even scalar $\eta$ that could be either heavier or lighter than the SM-like Higgs. By taking into account all theoretical and experimental constraints such as perturbativity, unitarity, vacuum stability, non-SM Higgs decays, $\mathrm{DM}$ direct detection, and $M$ relic density, we found viable $\mathrm{DM}$ is possible in the range from $\mathrm{GeV}$ to $\mathrm{TeV}$. Within the viable parameters space, both of the triple Higgs coupling and the di-Higgs production at LHC14 could be enhanced or reduced depending on the scalar mixing and the mass of the scalar particle $\eta$.
\end{abstract}

DOI: 10.1103/PhysRevD.104.075022

\section{INTRODUCTION}

It is a fact that $27 \%$ of the matter in the universe is made out of cold dark matter. Historically, its existence was proposed as a possible explanation for several astrophysical observations in the cluster of galaxies [1]. The combined analysis of the Planck satellite 2018 results gives a a value of the relic abundance of DM density [2]

$$
\Omega_{\mathrm{DM}} h^{2}=0.120 \pm 0.001
$$

where $h$ is the reduced Hubble constant and $\Omega_{\mathrm{DM}}$ denotes the DM energy density in unit of the critical density. Obviously, the DM candidate must be a stable particle, or at least its lifetime is much larger than the lifetime of the universe, with no direct interaction with the electroweak and strong forces. Its stability can be guaranteed by

\footnotetext{
*baouche.nabil@gmail.com

†ahriche@sharjah.ac.ae

"gaberfaisel@sdu.edu.tr

snasri@uaeu.ac.ae
}

Published by the American Physical Society under the terms of the Creative Commons Attribution 4.0 International license. Further distribution of this work must maintain attribution to the author(s) and the published article's title, journal citation, and DOI. Funded by SCOAP. imposing an appropriate symmetry, which can be discrete or continuous. In addition, it has also to be nonrelativistic, i.e., cold, as the possibility of hot DM is ruled out by several observations. Among these observations one can list briefly the pattern of fluctuations in the cosmic microwave background, the so-early formation of stars, galaxies, and clusters of galaxies, and the weak lensing signals we observe. Thus, one should consider going beyond Standard Model (BSM) physics for possible DM candidates. In the literature, many extensions of the SM have been proposed with the DM being a scalar [3], a fermion [4], or a vector boson [5-7].

In the literature, there are many vector DM models with different mass ranges and different annihilation. For example, the author of [6] proposed a vector DM model based on a hidden gauge symmetry $\left[\mathrm{SU}(2)_{\mathrm{HS}}\right]$, where the DM interacts with the SM particles only via mixing between the $\mathrm{SU}(2)_{\mathrm{HS}}$ doublet and the Higgs doublet. In [8], a similar model is proposed which is based on the same hidden symmetry where the DM interacts with the SM occurs via two hidden triplets, rather than one hidden doublet as in [6].

In this work, we consider the model [6] where the spin-1 gauge bosons couple to the SM through the Higgs portal. It has the privilege that the stability of the DM particles is guaranteed by the custodial symmetry associated with the gauge symmetry and particle content of the model. This symmetry is favored as one, in this case, does not need to 
impose any kind of discrete or global symmetry by hand. However, several important features of the model were not investigated due to the nonobservation of the Higgs boson at the time of the study. For instance, the measurements of some of the Higgs couplings to a reasonable precision can be used to constrain any heavy scalar state which mixes with the SM-like Higgs boson which will be carried out in this work. Additionally, triple Higgs coupling and the diHiggs production turn out to be so important to shed light on new physics and to understand the electroweak symmetry breaking. All these issues will be investigated in this work. Moreover, we provide the complete explicit analytic expressions of the cross section of the DM annihilation and coannihilation for different channels that contribute to the thermal relic density at freeze-out needed to estimate the DM relic density. These exact formulas were not reported in [6]. Finally, we will report the results related to the branching ratios of several decay modes of the scalars in the model which can be tested in future collider experiments.

The paper is organized as follows. First, we review the model proposed in [6] and discuss the mass eigenstates and their couplings that arise from the scalar potential in Sec. II. Then, in Sec. III, we investigate the theoretical and experimental constraints, such as vacuum stability, unitarity, and DM direct detection (DD) bound, that can be imposed on the model parameters. In Sec. IV, we consider the lightest gauge vector of the $\mathrm{SU}(2)_{\mathrm{HS}}$ to be a $\mathrm{DM}$ candidate and estimate its relic density by considering all possible allowed annihilation channels as well as the coannihilation effects. Next, we carry out a detailed study of the collider phenomenology of the model in Sec. V. Finally, we summarize our results and conclude in Sec. VI. Some relevant formulas and expressions of the effective potential and the cross-section contributions are given in the Appendix A and Appendix B, respectively.

\section{MODEL}

In this study, we consider the proposed model in [6]. The model, based on enlarging the gauge symmetry of the SM to include a non-Abelian gauge symmetry, is referred to as $\mathrm{SU}(2)_{\mathrm{HS}}$, under which all SM particles are singlets. The scalar sector of the model contains a new doublet $\phi$ that is charged under the group $\mathrm{SU}(2)_{\mathrm{HS}}$ and is singlet under the SM gauge group. The extra gauge bosons, associated with $\mathrm{SU}(2)_{\mathrm{HS}}$, are denoted by $A^{\mu}$. They couple to the SM only through the Higgs portal $\left(-\lambda_{m} \phi^{\dagger} \phi H^{\dagger} H\right)$ of $\mathrm{SU}(2)_{\mathrm{HS}}$ [6]. The Lagrangian of the model can be expressed as

$$
\begin{aligned}
\mathcal{L} \supset & -\frac{1}{4} F^{\mu \nu} \cdot F_{\mu \nu}+\left(D_{\mu} \phi\right)^{\dagger}\left(D^{\mu} \phi\right)-\lambda_{m} \phi^{\dagger} \phi H^{\dagger} H \\
& -\mu_{\phi}^{2} \phi^{\dagger} \phi-\lambda_{\phi}\left(\phi^{\dagger} \phi\right)^{2},
\end{aligned}
$$

where $D^{\mu} \phi=\left(\partial^{\mu}-i g_{\phi} \frac{\tau_{i}}{2} A_{i}^{\mu}\right) \phi$ with $g_{\phi}$ is the $\mathrm{SU}(2)_{\mathrm{HS}}$ gauge coupling, and $\tau_{i}$ are the Pauli matrices. The Higgs potential of the SM is parametrized as $\mathcal{L}^{\mathrm{SM}} \supset-\mu^{2} H^{\dagger} H-$ $\lambda\left(H^{\dagger} H\right)^{2}$ with $H^{T}=\left(\chi^{+}, \frac{1}{\sqrt{2}}\left[h^{\prime}+i \chi^{0}\right]\right)$. For suitable choice of the parameters $\mu_{\phi}^{2}$ and $\lambda_{m}$, the gauge symmetry $\mathrm{SU}(2)_{\mathrm{HS}}$ is spontaneously broken when the vacuum expectation value of $\phi, v_{\phi}$, is not vanishing. After expressing the new doublet in the unitary gauge as

$$
\phi=\frac{v_{\phi}+\eta^{\prime}}{\sqrt{2}} \exp \left\{-i \tau_{k} \frac{\xi_{k}}{v_{\phi}}\right\}\left(\begin{array}{l}
0 \\
1
\end{array}\right)
$$

one gets

$$
\begin{aligned}
\mathcal{L}= & \mathcal{L}_{\mathrm{SM}}-\frac{1}{4} F_{\mu \nu} \cdot F^{\mu \nu}+\frac{1}{8}\left(g_{\phi} v_{\phi}\right)^{2} A_{\mu} \cdot A^{\mu} \\
& +\frac{1}{8} g_{\phi}^{2} A_{\mu} \cdot A^{\mu} \eta^{\prime 2}+\frac{1}{4} g_{\phi}^{2} v_{\phi} A_{\mu} \cdot A^{\mu} \eta^{\prime} \\
& +\frac{1}{2}\left(\partial_{\mu} \eta^{\prime}\right)^{2}-\frac{\lambda_{m}}{2}\left(\eta^{\prime}+v_{\phi}\right)^{2} H^{\dagger} H-\frac{\mu_{\phi}^{2}}{2}\left(\eta^{\prime}+v_{\phi}\right)^{2} \\
& -\frac{\lambda_{\phi}}{4}\left(\eta^{\prime}+v_{\phi}\right)^{4},
\end{aligned}
$$

where $A_{\mu}=U A_{\mu}^{\prime} U^{-1}-\frac{i}{g_{\phi}}\left[\partial_{\mu} U\right] U^{-1}$ with $U=\exp \left\{-i \tau_{k} \xi_{k} /\right.$ $\left.v_{\phi}\right\} \in \mathrm{SU}(2)_{\mathrm{HS}}$. In the $A_{i}^{\mu}$ component space, the Lagrangian $\mathcal{L}$ in (4) has $\mathrm{SO}(3)$ custodial symmetry. ${ }^{1}$ As a consequence, the three $A_{i}^{\mu}$ components are degenerate in mass, $m_{A}=g_{\phi} v_{\phi} / 2$, stable, and hence can serve as vector DM candidates.

In order to proceed, we need to express $\mathcal{L}$ in (4) in terms of the mass eigenstates. This can be done after minimizing the scalar potential along both $\phi$ and $H$ directions and setting $H=\exp \left\{-i \tau_{k} \chi_{k} / v\right\} \cdot\left(0, \frac{1}{\sqrt{2}}\left[v+h^{\prime}\right]\right)^{T}$, where $v=$ $246 \mathrm{GeV}$ is the usual Higgs vacuum expectation value. By imposing the tadpole conditions $\partial V / \partial h=\partial V / \partial \eta=0$ one finds

$$
\mu^{2}=-\lambda v^{2}-\frac{1}{2} \lambda_{m} v_{\phi}^{2}, \quad \mu_{\phi}^{2}=-\lambda_{\phi} v_{\phi}^{2}-\frac{1}{2} \lambda_{m} v^{2} .
$$

The $h^{\prime}-\eta^{\prime}$ mixing due to the presence of the term of $\lambda_{m}$ in (4) leads to the mass-squared matrix

$$
M^{2}=\left(\begin{array}{cc}
2 \lambda v^{2} & \lambda_{m} v v_{\phi} \\
\lambda_{m} v v_{\phi} & 2 \lambda_{\phi} v_{\phi}^{2}
\end{array}\right),
$$

which gives the eigenvalues and the mixing

\footnotetext{
${ }^{1}$ Note that this custodial symmetry holds at all loop orders, but it is possible to break it by higher-dimension operators suppressed by a mass scale of order of the grand unification energy scale. This breaking can still lead to a cosmologically stable DM with interesting signatures at the indirect DM searches [9].
} 


$$
\begin{aligned}
m_{1,2}^{2} & =\lambda v^{2}+\lambda_{\phi} v_{\phi}^{2} \mp \sqrt{\left(\lambda v^{2}-\lambda_{\phi} v_{\phi}^{2}\right)^{2}+\lambda_{m}^{2} v^{2} v_{\phi}^{2}}, \\
t_{2 \beta} & =\frac{\lambda_{m} v v_{\phi}}{\lambda_{\phi} v_{\phi}^{2}-\lambda v^{2}}
\end{aligned}
$$

where $t_{2 \beta}=\tan 2 \beta$. By diagonalizing $M^{2}$, we get the mass eigenstates $h$ and $\eta$ that are defined as

$$
\left(\begin{array}{l}
h \\
\eta
\end{array}\right)=\left(\begin{array}{cc}
c_{\beta} & -s_{\beta} \\
s_{\beta} & c_{\beta}
\end{array}\right)\left(\begin{array}{l}
h^{\prime} \\
\eta^{\prime}
\end{array}\right),
$$

with $c_{\beta}=\cos \beta, s_{\beta}=\sin \beta$. Since the couplings are real, the eigenvalues of the matrix $M^{2}$ are required to be positive definite only if

$$
\lambda>0, \quad \lambda_{\phi}>0, \quad 2 \lambda_{m}+\sqrt{\lambda \lambda_{\phi}}>0 .
$$

In our analysis, we identify the $m_{h} \sim 125-\mathrm{GeV}$ eigenstate to be the SM-like Higgs boson and $\eta$ the other eigenstate; therefore, we have two cases where the SM-like Higgs eigenstate is the (1) light or the (2) heavy eigenstate. Then, in the first case, the $C P$-even scalar masses (7) should be written as

$$
m_{h, \eta}^{2}=\lambda v^{2}+\lambda_{\phi} v_{\phi}^{2} \mp\left(\lambda_{\phi} v_{\phi}^{2}-\lambda v^{2}\right) / c_{2 \beta}
$$

with $c_{2 \beta}=\cos 2 \beta$ and the quartic couplings can be expressed as

$$
\begin{aligned}
\lambda & =\frac{m_{h}^{2}}{2 v^{2}} c_{\beta}^{2}+\frac{m_{\eta}^{2}}{2 v^{2}} s_{\beta}^{2}, \quad \lambda_{\phi}=\frac{m_{h}^{2}}{2 v_{\phi}^{2}} s_{\beta}^{2}+\frac{m_{\eta}^{2}}{2 v_{\phi}^{2}} c_{\beta}^{2}, \\
\lambda_{m} & =\frac{s_{2 \beta}}{2 v v_{\phi}}\left(m_{\eta}^{2}-m_{h}^{2}\right) .
\end{aligned}
$$

In the second case, the $C P$-even scalar masses are given by (7) as

$$
m_{h, \eta}^{2}=\lambda v^{2}+\lambda_{\phi} v_{\phi}^{2} \pm\left(\lambda_{\phi} v_{\phi}^{2}-\lambda v^{2}\right) / c_{2 \beta}
$$

and the quartic couplings

$$
\begin{aligned}
\lambda & =\frac{m_{h}^{2}}{2 v^{2}} s_{\beta}^{2}+\frac{m_{\eta}^{2}}{2 v^{2}} c_{\beta}^{2}, \quad \lambda_{\phi}=\frac{m_{h}^{2}}{2 v_{\phi}^{2}} c_{\beta}^{2}+\frac{m_{\eta}^{2}}{2 v_{\phi}^{2}} s_{\beta}^{2}, \\
\lambda_{m} & =\frac{s_{2 \beta}}{2 v v_{\phi}}\left(m_{h}^{2}-m_{\eta}^{2}\right) .
\end{aligned}
$$

Clearly, the model can be described by the four free parameters $g_{\phi}, \lambda_{\phi}, \mu_{\phi}$, and $\lambda_{m}$, or equivalently by $s_{\beta}, g_{\phi}, m_{\eta}$, and $v_{\phi}$, in addition to the SM parameters.

Keeping only terms relevant to our study, in the Lagrangian in the mass eigenstates basis, we can write

$$
\begin{aligned}
\mathcal{L} \supset & -\frac{1}{4} F_{\mu \nu} \cdot F^{\mu \nu}+\frac{1}{2} m_{A}^{2} A_{\mu} \cdot A^{\mu}-\frac{1}{2} m_{\eta}^{2} \eta^{2}-\frac{1}{2} m_{h}^{2} h^{2}-\left(h c_{\beta}+\eta s_{\beta}\right)\left(\sum_{f} \frac{m_{f}}{v} f \bar{f}\right) \\
+ & {\left[\frac{s_{\beta}^{2}}{2 v} \eta^{2}+\frac{c_{\beta}^{2}}{2 v} h^{2}+\frac{s_{2 \beta}}{2 v} \eta h+\eta s_{\beta}+h c_{\beta}\right]\left(\frac{2 m_{W}^{2}}{v} W_{\mu}^{+} W^{-\mu}+\frac{m_{Z}^{2}}{v} Z^{\mu} Z_{\mu}\right) } \\
& +\left[\frac{c_{\beta}^{2}}{2 v_{\phi}} \eta^{2}+\frac{s_{\beta}^{2}}{2 v_{\phi}} h^{2}-\frac{s_{2 \beta}}{2 v_{\phi}} \eta h+\eta c_{\beta}-h s_{\beta}\right]\left(\frac{m_{A}^{2}}{v_{\phi}} A_{\mu} \cdot A^{\mu}\right) \\
& -\frac{1}{6} \rho_{h} h^{3}-\frac{1}{6} \rho_{\eta} \eta^{3}-\frac{1}{2} \rho_{1} \eta^{2} h-\frac{1}{2} \rho_{2} h^{2} \eta,
\end{aligned}
$$

where $f$ denotes the SM fermions and the $\rho$ 's parameters represent the $C P$-even scalar triple couplings that are given by

$$
\begin{aligned}
& \rho_{1}=-6 \lambda_{\phi} v_{\phi} c_{\beta}^{2} s_{\beta}+6 \lambda v s_{\beta}^{2} c_{\beta}+\lambda_{m}\left(v c_{\beta}^{3}-v_{\phi} s_{\beta}^{3}-2 v c_{\beta} s_{\beta}^{2}+2 v_{\phi} c_{\beta}^{2} s_{\beta}\right), \\
& \rho_{2}=6 \lambda_{\phi} v_{\phi} s_{\beta}^{2} c_{\beta}+6 \lambda v c_{\beta}^{2} s_{\beta}+\lambda_{m}\left(v s_{\beta}^{3}+v_{\phi} c_{\beta}^{3}-2 v c_{\beta}^{2} s_{\beta}-2 v_{\phi} s_{\beta}^{2} c_{\beta}\right), \\
& \rho_{\eta}=6 \lambda_{\phi} v_{\phi} c_{\beta}^{3}+6 \lambda v s_{\beta}^{3}+3 \lambda_{m} c_{\beta} s_{\beta}\left(v c_{\beta}+v_{\phi} s_{\beta}\right), \\
& \rho_{h}=-6 \lambda_{\phi} v_{\phi} s_{\beta}^{3}+6 \lambda v c_{\beta}^{3}+3 \lambda_{m} c_{\beta} s_{\beta}\left(v s_{\beta}-v_{\phi} c_{\beta}\right) .
\end{aligned}
$$

We notice from (14) that the couplings of $h$ and $\eta$ to SM particles are weighted by $c_{\beta}$ and $s_{\beta}$, respectively. Moreover, in the first case the scalar $\eta$ can additionally decay into Higgs pairs if $m_{\eta}>2 m_{h}$ with the partial decay width

$$
\Gamma(\eta \rightarrow h h)=\Theta\left(m_{\eta}-2 m_{h}\right) \frac{\rho_{2}^{2}}{32 \pi m_{\eta}} \sqrt{1-4 \frac{m_{h}^{2}}{m_{\eta}^{2}}},
$$

and in the second case, the Higgs can decay into a pair of $\eta$ if $m_{h}>2 m_{\eta}$, in addition to their possible decay to $A_{i} A_{i}$. 


\section{THEORETICAL AND EXPERIMENTAL CONSTRAINTS}

This model is subject to a number of theoretical and experimental constraints such as vacuum stability, perturbativity, perturbative unitarity, electroweak precision tests (EWPT), and the constraints from the Higgs decay. For the EWPT, it is expected that the new physics contribution to the oblique parameters $(\Delta S$ and $\Delta T)$ is negligible since the scalar doublet $\eta$ is a singlet under the SM gauge group. Then, by considering the constraints from the Higgs signal strength $\mu_{\text {tot }} \geq 0.89$ at $95 \%$ CL [10], the $h-\eta$ mixing makes both $\Delta S$ and $\Delta T$ very tiny, and all the space parameters will be allowed by the EWPT. In what follows, we discuss the above-mentioned constraints in details.

(i) Unitarity constraints

Possible constraints on the quartic couplings $\lambda, \lambda_{\phi}$, and $\lambda_{m}$ can be derived upon requiring that the amplitudes for the scalar-scalar scattering $S_{1} S_{2} \rightarrow$ $S_{3} S_{4}$ at high energies respect the tree-level unitarity [11]. Here, $S_{i}$ can be $h$ or $\eta$ for $i=1,2,3,4$. This can be understood as, at high energies, the dominant contributions to these amplitudes are those mediated by the quartic couplings [12]. Denoting the eigenvalues of the scattering matrix as $\Lambda_{i}$, the unitarity condition reads

$$
\left|\Lambda_{i}\right| \leq 8 \pi
$$

In the model under concern the above bound results in the following constraints:

$$
\begin{aligned}
& \lambda_{m} \leq 8 \pi, \quad \lambda \leq 4 \pi, \quad \lambda_{\phi} \leq 4 \pi, \\
& 3\left(\lambda+\lambda_{\phi}\right) \pm \sqrt{9\left(\lambda-\lambda_{\phi}\right)^{2}+4 \lambda_{m}^{2}} \leq 8 \pi .
\end{aligned}
$$

(ii) Vacuum stability and perturbativity

The quartic couplings of the scalar potential are subjected to a number of constraints to ensure that the potential is bounded from below and that the couplings remain perturbative as well as the electroweak vacuum to be stable all the way up to the Planck scale. For the scalar potential to be bounded from below, the conditions $\lambda>0, \lambda_{\phi}>0$, and $\lambda \lambda_{\phi}>$ 0 must hold and for the case $\lambda_{m}<0$, the condition $2 \lambda_{m}+\sqrt{\lambda \lambda_{\phi}}>0$ must be also satisfied. Recall that one needs $4 \lambda \lambda_{\phi}>\lambda_{m}^{2}$ so that the Higgs mixing matrix $M^{2}$ is positive definite and thus $m_{h}, m_{\eta}>0$.

(iii) Constraints on the Higgs decays

Since the Higgs couplings are modified in our model, and since there exist new particles with new interactions, then the Higgs total decay width and branching ratios get modified. Here, all the vortices of the Higgs-gauge fields and Higgs fermions are scaled by $c_{\beta}$; therefore, the Higgs partial decay widths to the SM particles are scaled as $\Gamma\left(h \rightarrow X_{\mathrm{SM}} \bar{X}_{\mathrm{SM}}\right)=c_{\beta}^{2} \Gamma_{\mathrm{SM}}\left(h \rightarrow X_{\mathrm{SM}} \bar{X}_{\mathrm{SM}}\right)$. In addition to the SM final states, the Higgs may decay into new gauge bosons (dark photons) if kinematically allowed, with the Higgs partial decay width

$$
\begin{aligned}
& \Gamma_{\text {inv }}(h \rightarrow A A) \\
& =\sum_{i=1}^{3} \Gamma\left(h \rightarrow A_{i} A_{i}\right)=3 \Theta\left(m_{h}-2 m_{A}\right) \\
& \times \frac{g_{\phi}^{2} m_{h}^{3} s_{\beta}^{2}}{64 \pi m_{A}^{2}} \sqrt{1-4 \frac{m_{A}^{2}}{m_{h}^{2}}}\left\{1-4 \frac{m_{A}^{2}}{m_{h}^{2}}+12 \frac{m_{A}^{4}}{m_{h}^{4}}\right\} ;
\end{aligned}
$$

this channel is open when $m_{A}<m_{h} / 2$. The factor " 3 " in (19) refers to the summation over $A_{i} A_{i}$. In case 2 where the condition $m_{h}>2 m_{\eta}$ may be fulfilled, the decay channel $h \rightarrow \eta \eta$ is open and the partial width is given by

$$
\Gamma(h \rightarrow \eta \eta)=\Theta\left(m_{h}-2 m_{\eta}\right) \frac{\rho_{1}^{2}}{32 \pi m_{h}} \sqrt{1-4 \frac{m_{\eta}^{2}}{m_{h}^{2}}} .
$$

Therefore, the Higgs total decay width can be written as

$$
\Gamma_{h}=\Gamma_{\mathrm{fv}}+c_{\beta}^{2} \Gamma_{h}^{\mathrm{SM}},
$$

where $\Gamma_{h}^{\mathrm{SM}}=4.2 \mathrm{MeV}$ is the Higgs total decay width in the SM; $\Gamma_{\mathrm{BSM}}=\Gamma_{\text {inv }}$ for case 1 ; and $\Gamma_{\mathrm{BSM}}=\Gamma_{\text {inv }}+\Gamma_{\text {und }}$ for case2. Here, the undetermined Higgs decay width $\Gamma_{\text {und }}=\Gamma(h \rightarrow \eta \eta)$, which is different than the invisible one at collider, since the light scalar can be seen at detector via the decay to light fermions $\eta \rightarrow f \bar{f}$. These decays do not match the known SM ones; hence, the signal $h \rightarrow \eta \eta \rightarrow f_{1} \bar{f}_{1} f_{2} \bar{f}_{2}$ is named undetermined. Then, the invisible and undetermined branching ratio must respect the constraints $[13,14]^{2}$

$$
\mathcal{B}_{\text {inv }}<0.26, \quad \mathcal{B}_{\text {und }}<0.22, \quad \mathcal{B}_{\text {inv }}+\mathcal{B}_{\text {und }} \leq 0.47
$$

In addition, the Higgs total decay width should lie in the range $[17,18]$

$$
1.0 \mathrm{MeV}<\Gamma_{h}<6.0 \mathrm{MeV} .
$$

\footnotetext{
${ }^{2}$ Recent measurements by ATLAS [15] and CMS [16] of the invisible decay width of the SM Higgs boson give $\mathcal{B}_{\text {inv }}<0.11$ and $\mathcal{B}_{\text {inv }}<0.19$, respectively. In our numerical scan, we will consider the recent ATLAS bound.
} 
(iv) DM direct detection constraints

The DM candidate can interact with nucleons via $t$-channel diagrams that are mediated by $\mathrm{h}$ or $\eta$ as shown in Fig. 1.

Measuring nuclear recoil energy resulting from the elastic scattering of DM particle off nucleus in detector can serve as a direct search of DM particles. The results of such searches can be used to impose constraints on the relevant parameters of the model. At tree level, the spin-independent elastic scattering of the vector DM off nucleus, mediated by $h$ or $\eta$ exchange, given in [6], can be simplified as

$$
\begin{aligned}
\sigma_{S I}(N A \rightarrow N A) & =\frac{1}{64 \pi} f^{2} g_{\phi}^{4} s_{2 \beta}^{2} m_{N}^{2} \frac{v_{\phi}^{2}}{v^{2}} \frac{\left(m_{h}^{2}-m_{\eta}^{2}\right)^{2}}{m_{h}^{4} m_{\eta}^{4}}\left(\frac{m_{N}}{m_{N}+m_{A}}\right)^{2} \\
& =6.45765608 \times 10^{-42} \mathrm{~cm}^{2} \mathrm{~s}_{2 \beta}^{2}\left(\frac{g_{\phi}}{0.5}\right)^{4}\left(\frac{v_{\phi}}{100 \mathrm{GeV}}\right)^{2}\left(\frac{m_{h}}{m_{\eta}}\right)^{4}\left(1-\frac{m_{\eta}^{2}}{m_{h}^{2}}\right)^{2}\left(1+\frac{m_{A}}{m_{N}}\right)^{-2} .
\end{aligned}
$$

Here, $m_{N}$ denotes the nucleon mass and $f=0.3$ [6] parametrizes the Higgs nucleon coupling. This has to be compared with the present experimental upper bound on this cross section [19].

(v) Renormalization group equation

The constraints from vacuum stability and couplings perturbativity can be determined by the renormalization group evolution of $\lambda, \lambda_{m}, \lambda_{\phi}$. At one-loop $\beta$ functions and upon neglecting all the Yukawa couplings except for $y_{t}$, the relevant equations are given as $[10,20]$

$$
\begin{aligned}
16 \pi^{2} d \lambda / d t & =24 \lambda^{2}+2 \lambda_{m}^{2}-6 y_{t}^{4}+\lambda\left(12 y_{t}^{2}-\frac{9}{5} g_{1}^{2}-9 g_{2}^{2}\right)+\frac{27}{200} g_{1}^{4}+\frac{9}{20} g_{1}^{2} g_{2}^{2}+\frac{9}{8} g_{2}^{4}, \\
16 \pi^{2} d \lambda_{\phi} / d t & =24 \lambda_{\phi}^{2}+2 \lambda_{m}^{2}-9 \lambda_{\phi} g_{\phi}^{2}+\frac{9}{4} g_{\phi}^{4}, \\
16 \pi^{2} d \lambda_{m} / d t & =\lambda_{m}\left(6 y_{t}^{2}+12 \lambda+12 \lambda_{\phi}+4 \lambda_{m}-\frac{9}{10} g_{1}^{2}-\frac{9}{2} g_{2}^{2}-\frac{9}{2} g_{\phi}^{2}\right), \\
16 \pi^{2} d g_{i} / d t & =b_{i} g_{i}^{3} \quad \text { with }\left(b_{1}, b_{2}, b_{3}, b_{\phi}\right)=(41 / 6,-19 / 6,-7,-43 / 6), \\
16 \pi^{2} d \lambda_{t} / d t & =y_{t}\left(\frac{9}{2} y_{t}^{2}-\frac{17}{20} g_{1}^{2}-\frac{9}{4} g_{2}^{2}-8 g_{3}^{2}\right),
\end{aligned}
$$

where $g_{i}=\left(g_{1}, g_{2}, g_{3}\right)$ represents the SM gauge couplings and $g_{\phi}$ is the gauge coupling of the new $\mathrm{SU}(2)_{H}$. In what follows, we will use (25) to check whether the conditions of the vacuum stability, perturbativity, and unitarity are fulfilled at higher scales: $\Lambda=100 \mathrm{TeV}, 10^{4} \mathrm{TeV}$, and $\Lambda=m_{\text {Planck }}$.

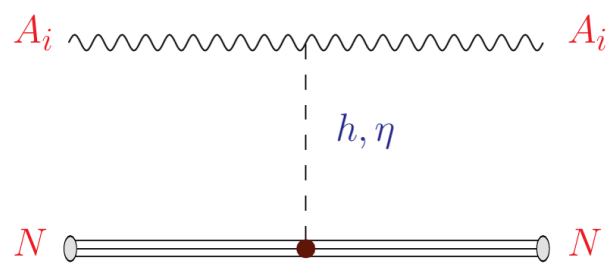

FIG. 1. Feynman diagrams that lead to DM direct detection at underground detectors.

\section{DARK MATTER RELIC DENSITY}

In order to estimate the relic density, one has to estimate the freeze-out temperature and the annihilation cross section. The thermal relic density at freeze-out is given in the terms of the thermally averaged annihilation cross section by [21]

$$
\Omega_{\mathrm{DM}} h^{2} \simeq \frac{1.04 \times 10^{9}}{M_{p l}} \frac{x_{F}}{\sqrt{g_{*}\left(x_{F}\right)}} \frac{3}{\left\langle\sigma(A A) v_{r}\right\rangle},
$$

where $v_{r}$ is the relative velocity, $M_{p l}=1.22 \times 10^{19} \mathrm{GeV}$ is the Planck mass, $g_{*}$ counts the total number of relativistic degrees of freedom, and $x_{F}=m_{A} / T_{f}$ is the inverse freezeout temperature. The factor " 3 " in (26) comes from the fact that the total relic density is the summation of the contributions of the three vector bosons $A_{i}$ that have the same masses, same interactions, and hence give the same 


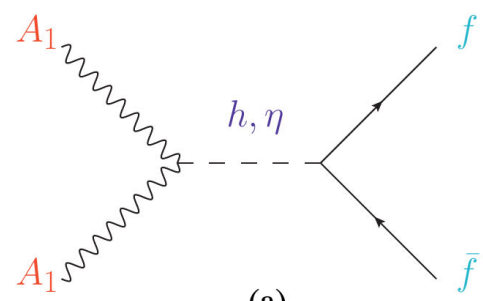

(a)

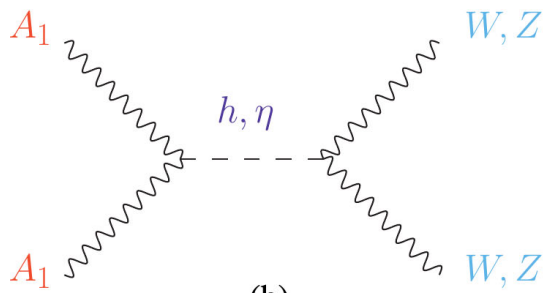

(b)

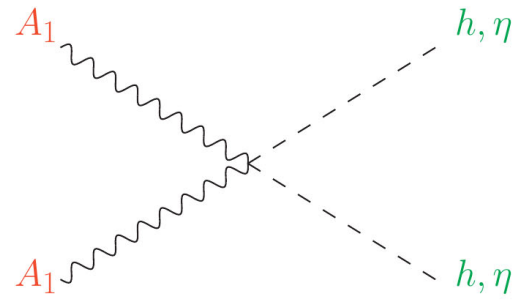

(d)

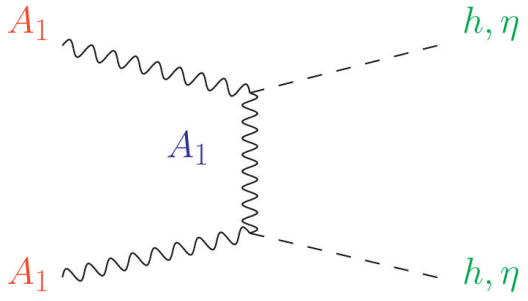

(e)

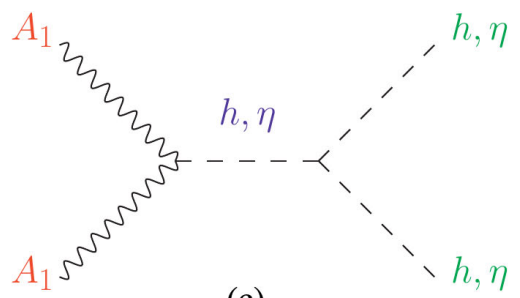

(c)

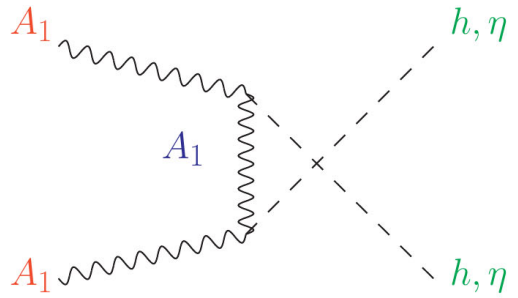

(f)

FIG. 2. Different DM self-annihilation channels.

contribution to the relic density. The total thermally averaged annihilation cross section

$$
\begin{aligned}
\left\langle\sigma(A A) v_{r}\right\rangle= & \frac{1}{8 T m_{A}^{4} K_{2}^{2}\left(m_{A} / T\right)} \sum_{X} \int_{4 m_{A}^{2}}^{\infty} d s \sigma_{A A \rightarrow X}(s) \sqrt{s} \\
& \times\left(s-4 m_{A}^{2}\right) K_{1}(\sqrt{s} / T)
\end{aligned}
$$

where $K_{1,2}$ are the modified Bessel functions and $\sigma_{A A \rightarrow X}(s)$ is the annihilation cross section due to the channel $A A \rightarrow X$, at the Center of Mass (CM) energy $\sqrt{s}$.

The freeze-out parameter $x_{F}$ can be obtained iteratively from the equation

$$
x_{F}=\log \left(\frac{5}{4} \sqrt{\frac{45}{8}} \frac{3}{2 \pi^{3}} \frac{M_{p l} m_{A}\left\langle\sigma(A A) v_{r}\right\rangle}{\sqrt{g_{*}\left(x_{F}\right) x_{F}}}\right),
$$

where the factor " 3 " in (28) refers to the DM degrees of freedom.

In case where the DM candidate is close in mass to other species, the coannihilation effect becomes important, and in order to take it into account, the thermal average cross section, $\left\langle\sigma(A A) v_{r}\right\rangle$, in (26) and (28) should be replaced with the effective one $\left\langle\sigma_{\mathrm{eff}}(A A) v_{r}\right\rangle$. The effective thermally averaged annihilation cross section is given by [21]

$$
\begin{aligned}
\sigma_{\mathrm{eff}}(x)= & \sum_{i j}^{N} \frac{g_{i} g_{j}}{g_{\mathrm{eff}}^{2}} \sigma_{i j}\left(1+\Delta_{i}\right)^{3 / 2}\left(1+\Delta_{j}\right)^{3 / 2} \\
& \times \exp \left[-x\left(\Delta_{i}+\Delta_{j}\right)\right] \\
g_{\mathrm{eff}}(x)= & \sum_{i=1}^{N} g_{i}\left(1+\Delta_{i}\right) \exp \left(-x \Delta_{i}\right)
\end{aligned}
$$

where $\Delta_{i}=\frac{m_{i}-m_{\mathrm{DM}}}{m_{\mathrm{DM}}}$ and $g_{i}$ is the multiplicity of the species " $i$ ". In our model, we consider the coannihilation effect coming from the mass degeneracy between the vector bosons $A_{i}$, and therefore $g_{i}=3, g_{\text {eff }}=9$, $\sigma\left(A_{i} A_{j \neq i}\right)=\sigma\left(A_{1} A_{2}\right)$, and $\sigma\left(A_{i} A_{i}\right)=\sigma\left(A_{1} A_{1}\right)$. Thus, the effective thermally averaged cross section reads

$$
\sigma_{\text {eff }}(A A)=\frac{1}{3}\left[\sigma\left(A_{1} A_{1} \rightarrow \text { all }\right)+2 \sigma\left(A_{1} A_{2} \rightarrow \text { all }\right)\right] .
$$

In our analysis, we estimate the relic density by considering (26), (28), and (30), and confront it with the recent precise measurements from the PLANCK satellite shown in (1). Here, we consider $3 \sigma$ range, i.e., $0.117 \leq \Omega_{\mathrm{DM}} h^{2} \leq 0.123$. In the rest of this section, we estimate the different contributions to the cross sections $\sigma\left(A_{1} A_{1}\right)$ and $\sigma\left(A_{1} A_{2}\right)$.

For the cross section $\sigma\left(A_{1} A_{1}\right)$, we have many channels: $f \bar{f}, W W, Z Z, h h, \eta \eta$, and $h \eta$ as shown in Fig. 2, while for the cross section $\sigma\left(A_{1} A_{2}\right)$, we have only one channel as shown in Fig. 3.

From the diagram in Fig. 2(a), the annihilation cross section into fermion pairs $(f \bar{f})$ at $\mathrm{CM}$ energy $\sqrt{s}$ is estimated to be

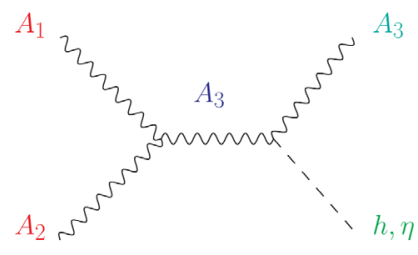

(a)

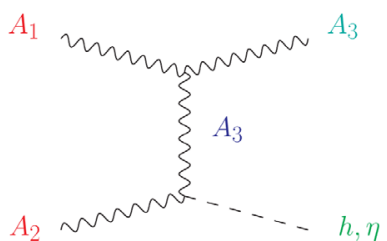

(b)
FIG. 3. The DM coannihilation channel. 


$$
\begin{aligned}
& \sigma\left(A_{1} A_{1} \rightarrow f \bar{f}\right) v_{r}= N_{c} \frac{s_{2 \beta}^{2}}{144 \pi} \frac{m_{A}^{4} m_{f}^{2}}{v_{\phi}^{2} v^{2}} \mathcal{B W}\left(1-\frac{4 m_{f}^{2}}{s}\right)^{\frac{3}{2}} \\
& \times\left(12-4 \frac{s}{m_{A}^{2}}+\frac{s^{2}}{m_{A}^{4}}\right), \\
& \mathcal{B W}=\left|\frac{i}{s-m_{h}^{2}+i m_{h} \Gamma_{h}}-\frac{i}{s-m_{\eta}^{2}+i m_{\eta} \Gamma_{\eta}}\right|^{2},
\end{aligned}
$$

with $s_{2 \beta}=\sin (2 \beta), N_{c}$ is the color factor ( 1 for leptons and 3 for quarks), and $\Gamma_{h}\left(\Gamma_{\eta}\right)$ is the total decay width of the Higgs (scalar $\eta$ ).

The annihilation cross section into gauge bosons $V=W$, $Z$ as shown in Fig. 2(b) is found at CM energy $\sqrt{s}$ to be

$$
\begin{aligned}
\sigma\left(A_{1} A_{1} \rightarrow V V\right) v_{r}= & \delta_{V} \frac{s_{2 \beta}^{2} m_{A}^{4} m_{V}^{4}}{288 \pi s v^{2} v_{\phi}^{2}} \mathcal{B W}\left(1-\frac{4 m_{V}^{2}}{s}\right)^{1 / 2} \\
& \times\left(12-4 \frac{s}{m_{A}^{2}}+\frac{s^{2}}{m_{A}^{4}}\right) \\
& \times\left(12-4 \frac{s}{m_{V}^{2}}+\frac{s^{2}}{m_{V}^{4}}\right)
\end{aligned}
$$

where $V$ stands for $W^{ \pm}$or $Z$ bosons; $\delta_{W}=2$ and $\delta_{Z}=1$.

In order to estimate the cross section for the processes $A_{1} A_{1} \rightarrow X \equiv h h, \eta \eta$, we estimate the amplitude by considering the diagrams in Fig. 2(c), Fig. 2(d), Fig. 2(e), and Fig. 2(f) and write its averaged squared amplitude in powers of $t-m_{A}^{2}$ and $u-m_{A}^{2}$ as

$$
\begin{aligned}
|\overline{\mathcal{M}}|^{2}= & \frac{1}{2}\left\{\frac{Q_{1} m_{A}^{4}}{\left(t-m_{A}^{2}\right)^{2}}+\frac{Q_{2} m_{A}^{2}}{t-m_{A}^{2}}+Q_{3}+\frac{Q_{4}}{m_{A}^{2}}\left(t-m_{A}^{2}\right)\right. \\
& \left.+\frac{Q_{5}}{m_{A}^{4}}\left(t-m_{A}^{2}\right)^{2}+\frac{Q_{6}}{m_{A}^{6}}\left(t-m_{A}^{2}\right)^{3}\right\}+\{t \rightarrow u\} .
\end{aligned}
$$

Then, the integration over the angles of the $t$ terms and $u$ terms in (34) leads to identical contributions, where the cross section can be presented as

$$
\begin{aligned}
\sigma v_{r}= & \frac{Q_{0}}{s}\left\{\frac{2 Q_{1}}{A^{2}-B^{2}}+\frac{Q_{2}}{B} \ln \left(\frac{A+B}{A-B}\right)+2 Q_{3}+2 Q_{4} A\right. \\
& \left.+\frac{2}{3} Q_{5}\left(3 A^{2}+B^{2}\right)+2 Q_{6} A\left(A^{2}+B^{2}\right)\right\},
\end{aligned}
$$

where the dimensionless parameters $Q_{i}, A$, and $B$ are given in Appendix B. For the processes $A_{1} A_{1} \rightarrow X \equiv h \eta$ [i.e., diagrams in Fig. 2(c), Fig. 2(d), and Fig. 2(e)] and $A_{1} A_{2} \rightarrow X \equiv A_{3} h, A_{3} \eta$ (i.e., diagrams in Fig. 3), the averaged squared amplitude can be written only in powers of $t-m_{A}^{2}$, i.e.,

$$
\begin{aligned}
|\overline{\mathcal{M}}|^{2}= & \frac{Q_{1} m_{A}^{4}}{\left(t-m_{A}^{2}\right)^{2}}+\frac{Q_{2} m_{A}^{2}}{t-m_{A}^{2}}+Q_{3}+\frac{Q_{4}}{m_{A}^{2}}\left(t-m_{A}^{2}\right) \\
& +\frac{Q_{5}}{m_{A}^{4}}\left(t-m_{A}^{2}\right)^{2}+\frac{Q_{6}}{m_{A}^{6}}\left(t-m_{A}^{2}\right)^{3}
\end{aligned}
$$

and after integration we get the cross section that has the form (35). The dimensionless parameters $Q_{i}, A$, and $B$ for the processes $A_{1} A_{1} \rightarrow X \equiv h \eta$ and $A_{1} A_{2} \rightarrow X \equiv A_{3} h, A_{3} \eta$ are also given in Appendix B.

Dark matter candidates gravitationally accumulated in astrophysics objects at the galactic and extragalactic scales can self-annihilate or decay to SM particles, producing signals of anomalous flux of photons, neutrinos, or charged cosmic rays. The observation of these type of signals serves as indirect detection of dark matter. Moreover, as shown in [6], an enhancement of the positron emission through Sommerfeld effects $[22,23]$ can be large enough to explain the excess of positrons observed by the Pamela experiment [24]. This possibility holds either for $m_{A}$ in the multi-TeV range through DM annihilation to the final states $h h, h \eta, \eta \eta$, or for values of $m_{A}$ larger than $\sim 100 \mathrm{GeV}$ with $m_{\eta} \sim$ $1 \mathrm{GeV}$ via DM annihilation to $\eta \eta, h \eta$ followed by $\eta$ decays predominantly to leptons. Allowing for the breaking of the custodial symmetry by nonrenormalizable dimension six operators suppressed by a scale of order $10^{16} \mathrm{GeV}$ will induce the decay of DM with a lifetime much larger than the age of the universe, resulting in a flux of stable particles, such as gamma rays, positrons, and antiprotons [9]. For instance, the tree level, the vector DM two-body decay to the final states $\gamma h$ or $\gamma \eta$ yields the gamma lines which could be observed by the Fermi-LAT experiment [25].

\section{COLLIDER PHENOMENOLOGY}

\section{A. Collider constraints and the parameters space}

In the model under consideration, we have only four free parameters. It is convenient to choose them as the mass of the DM, $m_{A}$, the mass of the new scalar, $m_{\eta}$, the gauge coupling $g_{\phi}$, and finally sine of the mixing angle $s_{\beta}$. In our analysis, we perform a numerical scan over the parameters space in the ranges given as

$\left|s_{\beta}\right| \leq 1, \quad g_{\phi} \leq \sqrt{4 \pi}, \quad m_{A}, \quad m_{\eta} \leq 3 \mathrm{TeV}$.

These ranges of the parameters space are subjected to the constraints of vacuum stabilty (9), perturbativity (at the weak scale), unitarity (18), Higgs decay (22) and (23), DM relic density (26), and DM direct detection (24), in addition to the collider constraints on the Higgs signal strength (40).

At the LHC, ATLAS and CMS experiments have observed the Higgs boson in several decay channels, mainly $h \rightarrow Z Z, W W, \gamma \gamma, \tau \tau, b \bar{b}$ [26]. The observation allowed both collaborations to measure some of the Higgs couplings to a reasonable precision [26]. In return, 
these measurements can be used to constrain any heavy scalar state which mixes with the SM-like Higgs boson. The desired constraints can be deduced using the data of the signal strength modifier $\mu_{X X}$ for a given search channel, $h \rightarrow X X$. The signal strength modifier is a measured experimental quantity for the combined production and decay and is defined as the ratio of the measured Higgs boson rate to its SM prediction $[10,26]$ :

$\mu_{X X}=\sigma(p p \rightarrow h \rightarrow X X) /\left.\sigma(p p \rightarrow h \rightarrow X X)\right|_{\mathrm{SM}}$.

In the narrow-width approximation, $\mu_{X X}$ takes the simple form as

$$
\mu_{X X}=\frac{\sigma(p p \rightarrow h) \times \mathcal{B}(h \rightarrow X X)}{\left.\sigma(p p \rightarrow h)\right|_{\mathrm{SM}} \times\left.\mathcal{B}(h \rightarrow X X)\right|_{\mathrm{SM}}} .
$$

As mentioned above, due to the Higgs mixing, the couplings of the observed $h_{125}$ boson to SM fermions and gauge bosons are modified with respect to the SM, by $c_{\beta}$. So, by considering the definitions of the Higgs decay width in (21), one can simplify signal strength modifier as

$$
\mu_{X X}=c_{\beta}^{2}\left(1-\mathcal{B}_{\mathrm{BSM}}\right),
$$

where $\mathcal{B}_{\mathrm{BSM}}=\mathcal{B}_{\text {inv }}$ for the case $m_{\eta}>m_{h} / 2$, and $\mathcal{B}_{\mathrm{BSM}}=$ $\mathcal{B}_{\text {inv }}+\mathcal{B}_{\text {und }}$ for $m_{\eta}<m_{h} / 2$. Thus, the measurement of $\mu_{X X}$ of the $h_{125}$ Higgs boson can be used to derive a strong constraint on the mixing angle $\beta$ and the exotic Higgs decay fraction. The constraints in (40) are complementary to those on the exotic Higgs decays shown in (22) and (23).

The ATLAS and CMS collaborations have presented the results of $\mu_{X X}$ for the various final states at the Run-I of the LHC. The LHC Run-I corresponds to $\sqrt{s}=7$ and $\sqrt{s}=$ $8 \mathrm{TeV}$ with the integrated luminosity $5 \mathrm{fb}^{-1}$ and $20 \mathrm{fb}^{-1}$, respectively. The combined results have been reported in [26]. Based on the reported result of the combined total signal strengths of the $h_{125}$ Higgs, with all production and decay channels combined, one obtains

$$
\mu_{\text {tot }} \geq 0.89 \text {, }
$$

at 95\% CL [10]. This can be translated into a bound on the Higgs mixing angle $\beta$ that reads $s_{\beta}^{2} \leq 0.11$ in the absence of exotic Higgs decays, i.e., $\mathcal{B}_{\mathrm{BSM}}=0$. Within the LHC Run-II at $\sqrt{s}=13 \mathrm{TeV}$, ATLAS and CMS collaborations reported more accurate results in some decay channels. For instances, observations at $5 \sigma$ have been achieved in the $b \bar{b}$ mode [27,28]. The combined results of $\mu_{X X}$ at $\sqrt{s}=$ $13 \mathrm{TeV}$ have been reported by CMS collaboration in [29], and by ATLAS collaboration in [13]. Unfortunately, a global combination of their obtained results for LHC Run-II was not yet performed. Finally, with more improvement of the experimental sensitivity on the Higgs signal strengths in the future, a more stringent bound $s_{\beta}<0.18$ is expected to be reached at the high-luminosity LHC in case of $\mathcal{B}_{\mathrm{BSM}}=0[30,31]$. For the scenario $m_{\eta}>m_{h}$, the results of the direct searches at the LHC, which have been performed by the ATLAS and CMS collaborations, of heavy Higgs decays into $Z Z, W W, \gamma \gamma, h h, t \bar{t}$ can set additional complementary constraints. In particular, the constraints will be imposed on $m_{\eta}$ and $\left|s_{\beta}\right|$. For detailed discussion about these constraints we refer to [10], where detailed study and analysis have been performed.

In what follows, we will show the results of our numerical scan by considering all the above aforementioned constraints. For instance, in Fig. 4, we present the viable parameters space among the intervals (37). In Fig. 5, the signal strength (39) is presented versus the total decay width of the new scalar $\left(\Gamma_{\eta}\right)$, and the DM observables are presented in Fig. 6.

In the top left, top right, and bottom left of Fig. 4, we show the viable $m_{\eta}-m_{A}$ space parameters, respecting the aforementioned constraints in the ranges listed in (37), where the palettes show the scalar mixing (top left), the new scalar vev $v_{\phi}$ (top-right), and the new gauge coupling $g_{\phi}$ (bottom left). One notices the existence of three distinct subregions in the $m_{\eta}-m_{A}$ plan, corresponding to (1) $m_{\eta}<m_{A}$, and it is the largest subregion, (2) $m_{\eta} \gtrsim 2 m_{A}$, and (3) $m_{A} \lesssim m_{h} / 2$. Moreover, we remark that the constraints imposed on the parameters space can be easily evaded for values of $m_{\eta}$ and $m_{A}$ close to or larger than the electroweak scale, mostly in the top-right region in each plot, for the preferred ranges of the other parameters given as $\left|s_{\beta}\right| \lesssim 0.1$, $v_{\phi} \gtrsim 10^{3}$ and large values of $g_{\phi} \gtrsim 0.1$. One has to mention that most of the nonviable parameters space (empty regions in Fig. 4, top right for example) are excluded mainly by DM direct detection and relic density requirements.

Recall that in setup, the new physics contribution to the oblique parameters $\Delta S$ and $\Delta T$ has negligible effect. As a consequence, having small values of $s_{\beta}$ [within the condition (41)] allows having values $m_{\eta}$ close to or larger than the electroweak scale without violating the constraints imposed on $\Delta S$ and $\Delta T$. On the other hand, DM direct detection constraints given in (24) can be respected for large values of DM masses. Concerning the DM mass, which is given by $m_{A}=g_{\phi} v_{\phi} / 2$, having small values of $g_{\phi}$ can be compensated by large values $v_{\phi}$ to get the desired values of $m_{A}$ that explain the parameters space in Fig. 4. One remarks that the scalar mixing is almost suppressed except in the regions around the degeneracies that are defined by the straight lines in Fig. 4 , i.e., $m_{\eta} \gtrsim 2 m_{A}$, $m_{A} \lesssim m_{h} / 2$, and $m_{\eta} \sim m_{h}$.

In Fig. 4, bottom right, we show the quartic coupling $\lambda_{\phi}$ versus $\lambda$ for the values of the coupling $\lambda_{m}$ shown in the palette. One notes that the constraints can be escaped when the mixing coupling $\lambda_{m}$ is small, preferably less than 0.5 , and the other quartic couplings $\lambda_{\phi}$ and $\lambda$ have values approximately greater than $10^{-3}$. This result is a consequence of applying 

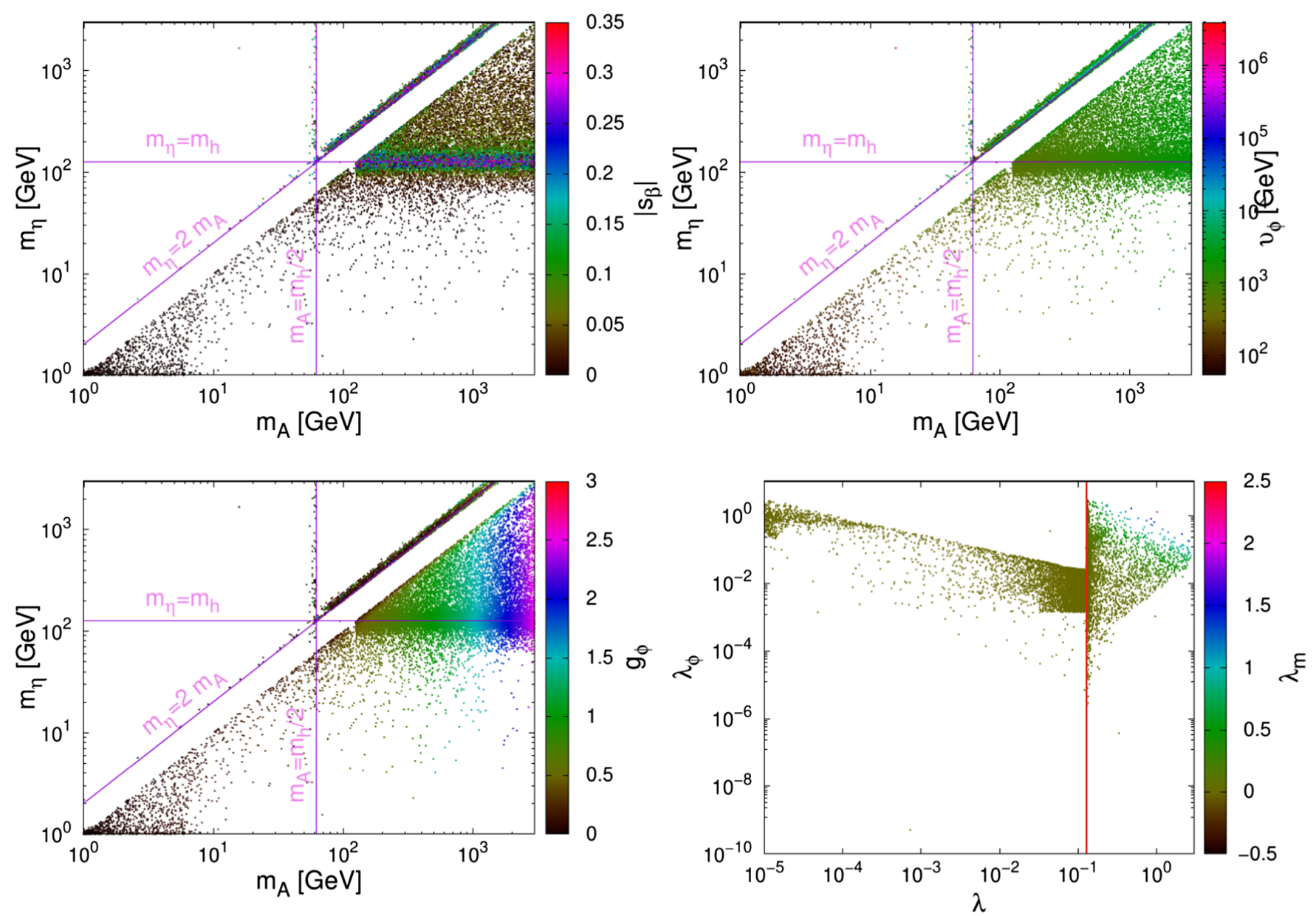

FIG. 4. The new scalar mass $m_{\eta}$ versus the DM mass $m_{A}$, where the palette shows the scalar mixing (top left), the new scalar vev (top right), and the new gauge coupling (bottom left). Bottom right: The quartic coupling $\lambda_{\phi}$ versus $\lambda$, where the coupling $\lambda_{m}$ is shown in the palette, where the red line represents the SM value of the Higgs quartic coupling $\lambda$. All the constraints mentioned in Sec. III are taken into account during the scan over the parameters space.

the unitarity constraints given by the last inequality in (18). Clearly, large values of both $\lambda_{\phi}$ and $\lambda$ should be accompanied by small values of $\lambda_{m}$ to satisfy the unitarity constraints.

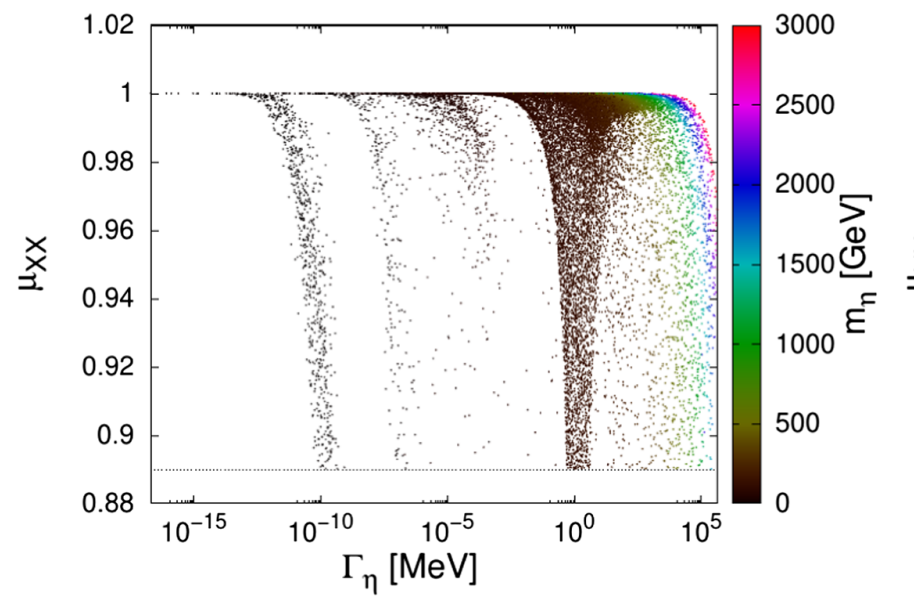

In Fig. 5, we show the signal strength (39) versus the total decay width of the new scalar $\left(\Gamma_{\eta}\right)$ where the palettes show the ranges of $m_{\eta}$ and $\Gamma_{\eta} / m_{\eta}$, respectively.

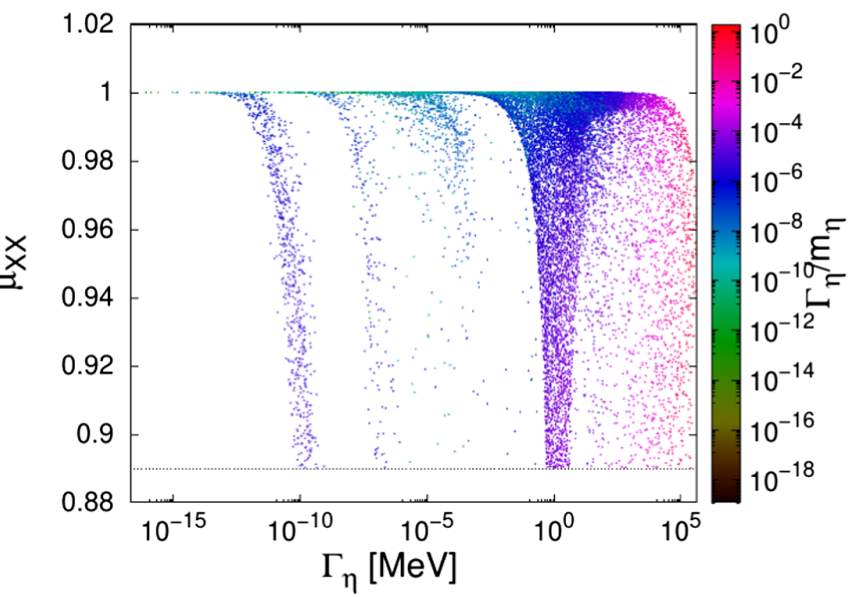

FIG. 5. The signal strength (39) versus the total decay width of the new scalar $\left(\Gamma_{\eta}\right)$. The palette shows the scalar mass $\left(m_{\eta}\right.$ in $\left.\mathrm{GeV}\right)$ on left side and the ratio $\Gamma_{\eta} / m_{\eta}$ on right side. 

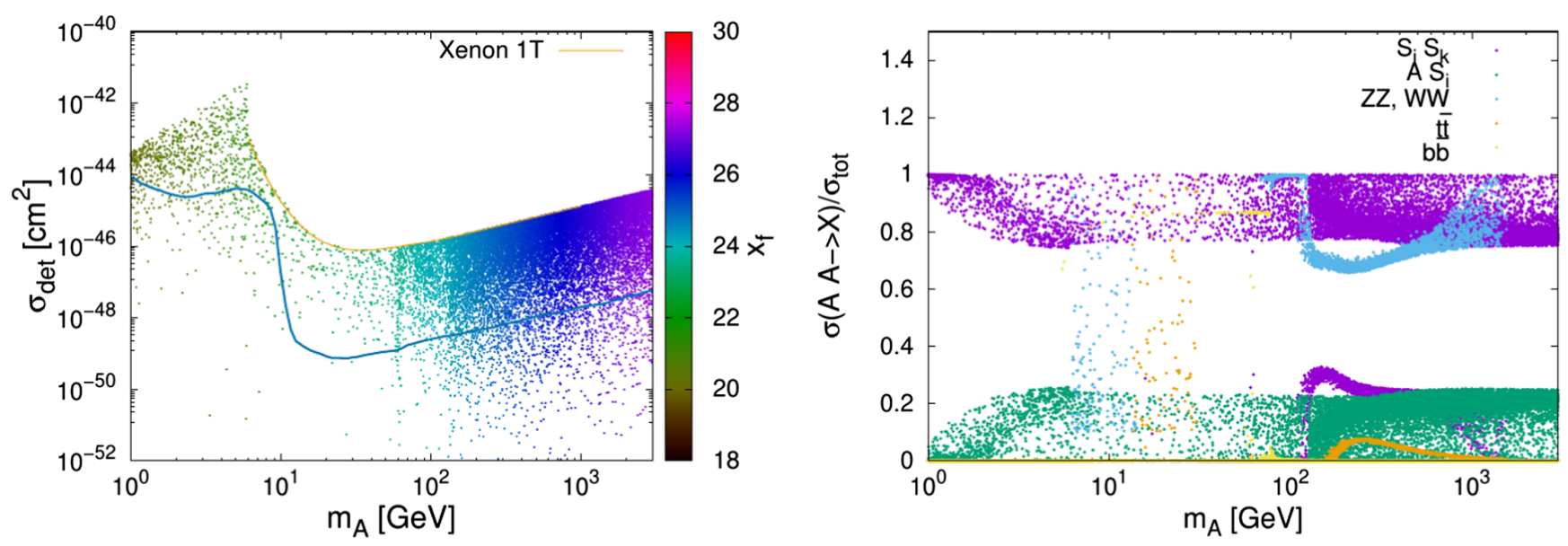

FIG. 6. Left: the DM direct detection cross section versus the DM mass, where the palette shows the freeze-out parameter $x_{f}=m_{A} / T_{f}$. The orange line represents the recent Xenon-1T bound [19], and the blue one represents the neutrino floor [32]. Right: the relative contributions to the thermal averaged cross section of different annihilation channels.

From (40), the signal strength has the value $c_{\beta}^{2}$ if the Higgs decays only to SM particles, i.e., $\mathcal{B}_{\mathrm{BSM}}=0$, which makes small mixing values the preferred ones as shown in Fig. 5, left. From Fig. 5, right, one notices that for $m_{\eta} \gtrsim 30 \mathrm{GeV}$, the total decay width has values $\Gamma_{\eta} \gtrsim 1 \mathrm{MeV}$, and it gets larger with large $m_{\eta}$ values. In Fig. 6, we show the DM-nucleon direct detection cross section (24) versus the DM mass (left) and the relative contribution of each annihilation channel to the total annihilation cross section at the freeze-out temperature (right).

From Fig. 6, left, one remarks that viable dark vector DM scenario is possible at all DM masses range, and future experiments such as Xenon $-n T$ [19] can probe a significant part of the parameters space. Indeed, some of the benchmark points cannot be probed since they are below the neutrino floor. The freeze-out parameter $x_{f}=m_{A} / T_{f}$ that is shown in the palette reads typical values $x_{f} \sim 17-28$. In order to figure out which of the DM annihilation channels is (are) efficient, we present in Fig. 6, right the relative contribution of each DM annihilation channel $(f \bar{f}$, $W W, Z Z, h h, \eta \eta$, and $h \eta$ ) to the total thermally averaged annihilation cross section at the freeze-out temperature $T_{f}=m_{A} / x_{f}$ versus the DM mass.

Clearly, from Fig. 6, right, one notes that the cross section tends to be dominated by the annihilation into scalar channels ( $h h, \eta \eta$, and $h \eta$ ) which are mediated by the exchanging of $h$, $\eta$, and $A_{i}$ bosons for all values of the DM mass. In addition the coannihilation contribution [i.e., the second term in (30)] could be large as $20 \%$ of the total thermally averaged annihilation cross section. The fact that the scalar channels $\eta \eta$ is also dominant for light $\mathrm{DM}[\mathcal{O}(\mathrm{GeV})]$ means that light DM implies light new scalar $\eta$. One has to mention also that the annihilation into gauge bosons could be efficient for DM masses around the $Z$ mass $\left(m_{Z}\right)$ and also around $\mathrm{TeV}$.

In Fig. 7, we present some branching ratios of decay modes of the Higgs (left), and the main branching ratios of

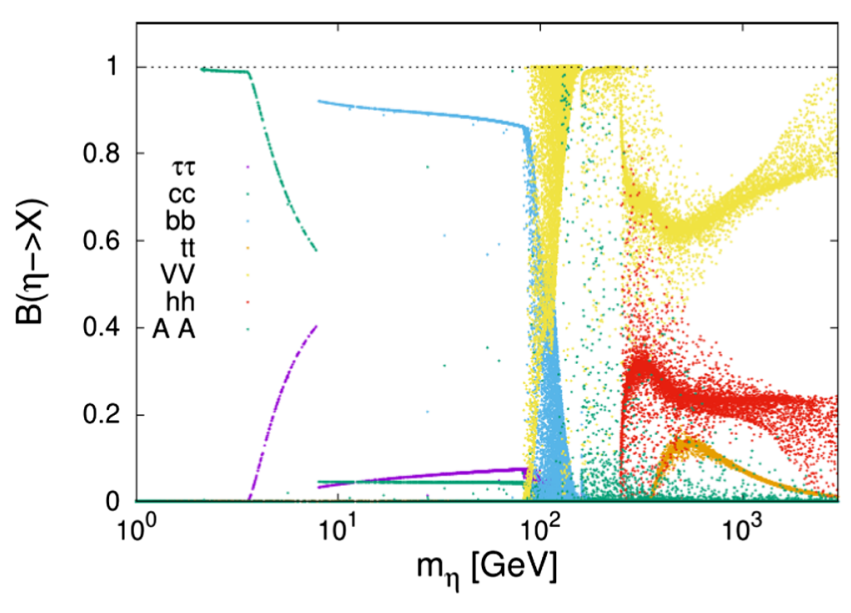

FIG. 7. Left: the Higgs branching ratios. Right: the branching ratios of the new scalar decay versus its mass. 
the scalar $\eta$ (right), especially $\gamma \gamma, A_{i} A_{i}, h h, t \bar{t}, b \bar{b}, V V$ as functions of $\eta$ mass.

One notices from Fig. 7, left, that the branching ratios of $h \rightarrow g g, h \rightarrow \gamma \gamma$, and $h \rightarrow \gamma Z$ have values close to the SM ones. Concerning the invisible and undetermined branching ratios ( $h \rightarrow A A$ and $h \rightarrow \eta \eta$ ), they can be significant within the allowed range for some of the parameters space. From Fig. 7, right, one can learn that scalar $\eta$ decay can be dominated by a specific contribution for some $m_{\eta}$ intervals. For instance, the decay into light quarks, mainly $\eta \rightarrow c \bar{c}$, dominates for the mass window $m_{\eta} \lesssim 8 \mathrm{GeV}$, while, it is dominated by $\eta \rightarrow b \bar{b}$ for $8 \mathrm{GeV} \lesssim m_{\eta} \lesssim 80 \mathrm{GeV}$. For the mass window $80 \mathrm{GeV} \lesssim m_{\eta} \lesssim 350 \mathrm{GeV}$ the decay will be dominated by $\eta \rightarrow W W, Z Z$ as well for some benchmark points with large scalar mass $m_{\eta}>2.5 \mathrm{TeV}$. The branching ratios of $\eta \rightarrow h h$ and $\eta \rightarrow t \bar{t}$ have maximal values of 0.35 and 0.18 , respectively, for scalar masses larger than $400 \mathrm{GeV}$. However, the invisible channel $\eta \rightarrow A A$ could be important for few benchmark points with mass between 100 and $350 \mathrm{GeV}$.
By running the Renormalization Group Equation (RGE) in (25) up to the high energy scales $\Lambda=100 \mathrm{TeV}$, $10^{4} \mathrm{TeV}$, and $\Lambda=m_{\text {Planck }}$, we obtain the running dimensionless scalar couplings. By imposing the conditions of perturbativity, vacuum stability, and unitarity at these energy scales, the parameters space get reduced as shown in Fig. 8, where the plots top left and bottom right in Fig. 4 are obtained after considering the above-mentioned conditions.

At higher scales, only benchmark with nonsuppressed $\lambda$ fulfills the above-mentioned conditions. In addition, only benchmark points with positive $\lambda_{m}$ are favored. The enhancement in $\lambda_{\phi}$ could be large by two orders of magnitude, which allows the benchmark points with small $\lambda_{\phi}$ values. For instance, for $20 \mathrm{k}$ benchmark points shown in Fig. 4 , the conditions of perturbativity, vacuum stability, and unitarity at the scales $\Lambda=100 \mathrm{TeV}, 10^{4} \mathrm{TeV}$, and $\Lambda=m_{\text {Planck }}$ are in the Higgs scalar potential, namely on the choice $\mu^{2}<0$ and $\lambda>0$. Hence, the partial experimental reconstruction of the Higgs scalar potential through
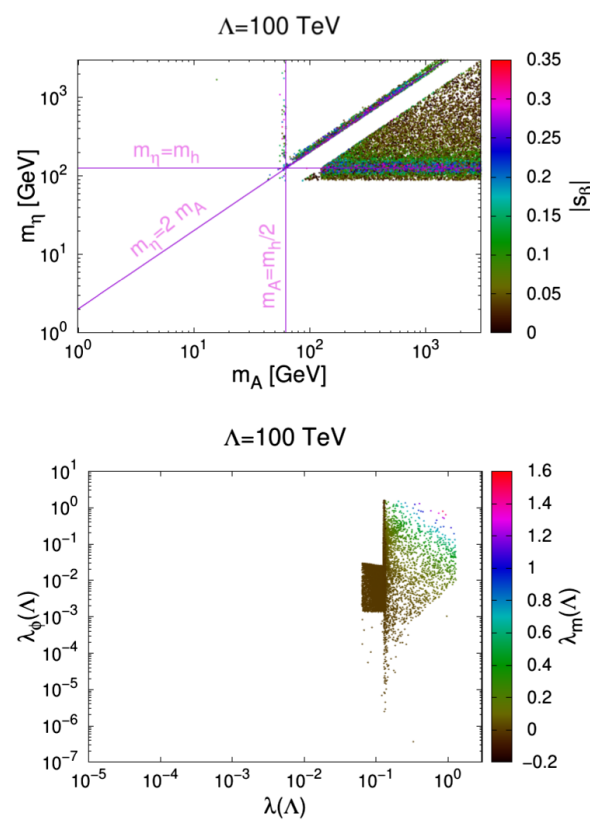

$\Lambda=100 \mathrm{TeV}$

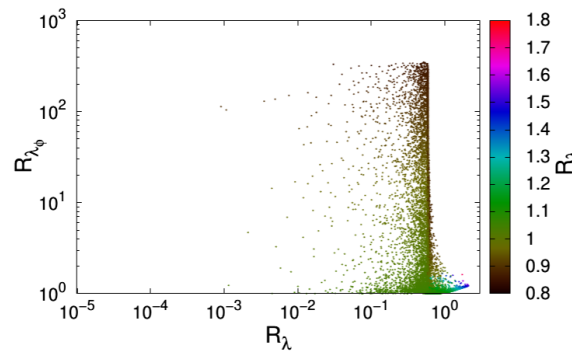

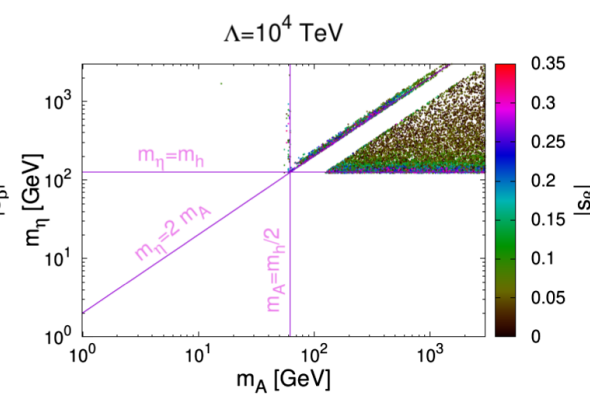
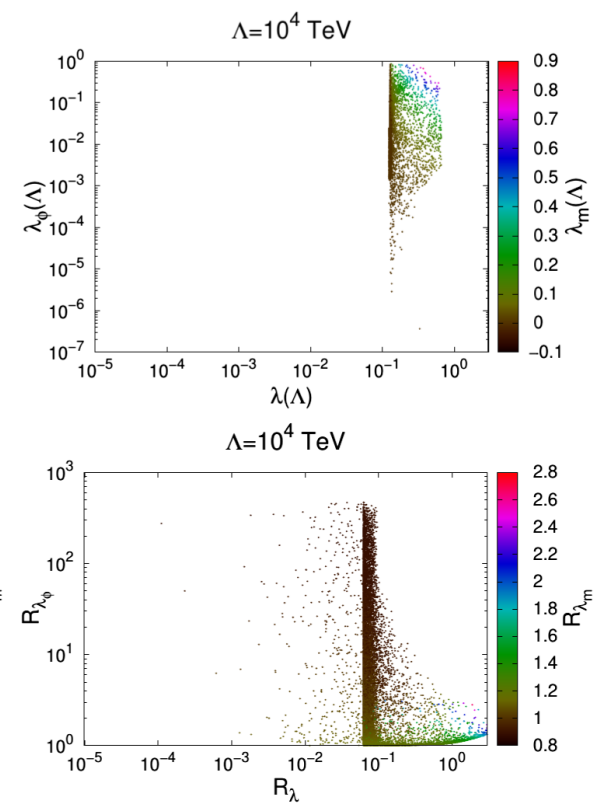
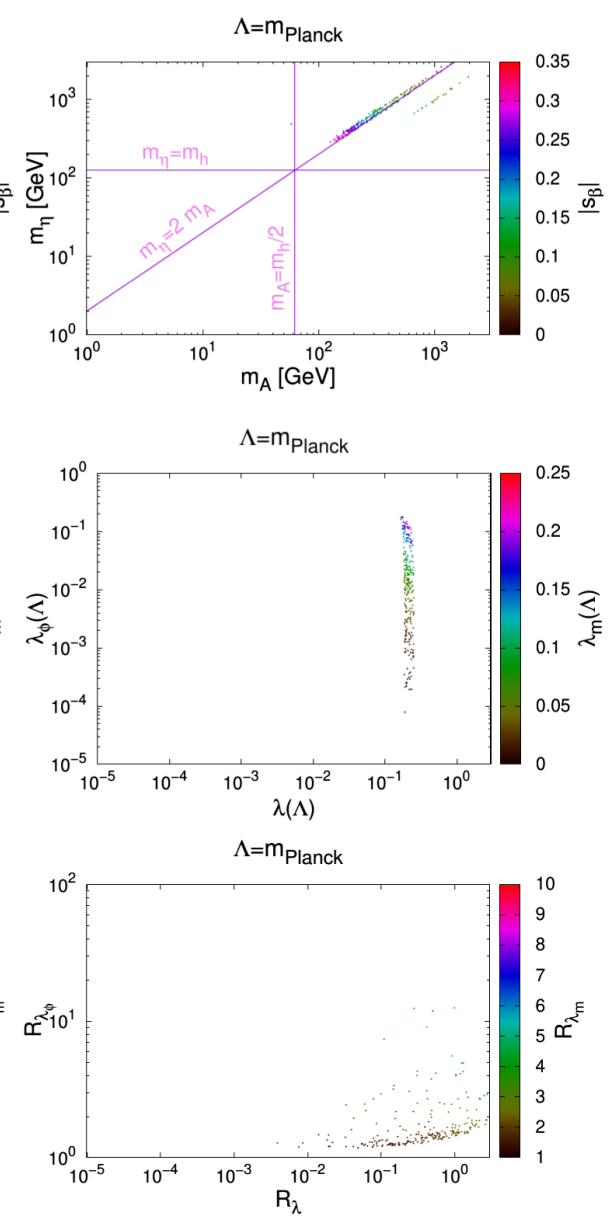

FIG. 8. Top: the updated version of Fig. 4, top left, after considering the aforementioned conditions (including the DM requirements) at scales $\Lambda=100 \mathrm{TeV}$ (left), $\Lambda=10^{4} \mathrm{TeV}$ (middle), and $\Lambda=m_{\text {Planck }}$ (right). Middle: the updated version of Fig. 4, bottom-right, within similar assumptions. Bottom: we show the coupling values at the running scale, and in the bottom ones, we show their relative enhancement $R_{\lambda_{i}}(\Lambda)=\lambda_{i}(\Lambda) / \lambda_{i}\left(m_{Z}\right)$. 
measuring the triple Higgs coupling, $\lambda_{h h h}$, turns out to be crucial to verify that the symmetry breaking is due to a SMlike Higgs sector [33]. Not only this, but it can also shed light on new physics [34] knowing that in many extensions of the SM, $\lambda_{h h h}$ can be modified by Higgs mixing effects or higher- order corrections induced by new particles, as we will show below. Consequently, the measurement of $\lambda_{h h h}$ is a crucial task in the LHC, although being challenging [33,35], and in future collider experiments.

Indirect probe of the triple Higgs coupling can be carried out through investigating the loop effects in some observables such as the single Higgs production [36-38], and the electroweak precision observables [39]. Using the $80 \mathrm{fb}^{-1}$ of LHC Run-II data, and upon the assumption that new physics can affect only $\lambda_{h h h}$, ATLAS collaboration set recently the bound $-1.5<\lambda_{h h h} / \lambda_{h h h}^{\mathrm{SM}}<6.7$ at $95 \%$ C.L. [40]. It should be noted that direct measurement of the triple Higgs coupling at the LHC is possible and can be achieved through the di-Higgs production. This production is dominated by the gluon-gluon fusion process. In the SM, the production has two main contributions originating from the triangle diagram induced by the triple Higgs coupling, and from the box diagram with the top quark running in the loop. As noted in [41], the two amplitudes, corresponding to the two contributions, interfere destructively. Consequently, at next-to-next-to-next-to-leading order $\left(\mathrm{N}^{3} \mathrm{LO}\right)$ and after including finite top quark mass effects, the estimated cross section at $14 \mathrm{TeV}$ LHC turns out to be small and equal to $38.65 \mathrm{fb}$ [41].

The analysis of the potential of measuring the di-Higgs production in the decay channels $b \bar{b} b \bar{b}, b \bar{b} \tau^{+} \tau^{-}, b \bar{b} W W^{*}$, $\gamma \gamma b \bar{b}, \gamma \gamma W W^{*}$, and $W W^{*} W W^{*}$ has been carried out in Refs. [33,35,42-56]. At $13 \mathrm{TeV}$ LHC with the luminosity of $36.1 \mathrm{fb}^{-1}$, the combination of the six analyses results in the constraint $-5<\lambda_{h h h} / \lambda_{h h h}^{\mathrm{SM}}<12$ at $95 \%$ C.L. [57]. It is expected that the sensitivity will be highly improved at the high-luminosity upgrade of the LHC (HL-LHC) [30] and future hadron colliders [58]. For instances, the future circular hadron collider, FCC- $h h$, with a center-of-mass energy of $100 \mathrm{TeV}$ and an integrated luminosity of $30 \mathrm{ab}^{-1}$ of data, will allow reaching a 5\% accuracy (at 68\% C.L.) on the measurement of the triple Higgs coupling [30,56,59].
The initial phase of the International Linear Collider (ILC) with a center-of-mass energy of $\sqrt{s}=250 \mathrm{GeV}$ cannot directly probe $\lambda_{h h h}$ via di-Higgs production [60]. However, this is not the case regarding single-Higgs production where an analysis using $2 \mathrm{ab}^{-1}$ of data can allow a measurement to $49 \%$ accuracy, at $68 \%$ C.L. [61]. Higher precision of $27 \%$ or $10 \%$, at $68 \%$ C.L., could possibly be reached using the data from ILC extensions to $500 \mathrm{GeV}\left(4 \mathrm{ab}^{-1}\right)$ or $1 \mathrm{TeV}\left(8 \mathrm{ab}^{-1}\right)$, respectively [62,63]. Moreover, at the same value of the confidence level, a higher accuracy of $0.93<\lambda_{h h h} / \lambda_{h h h}^{\mathrm{SM}}<1.11$ is possible to be reached using the combination of $1 \mathrm{ab}^{-1}$ of data at $380 \mathrm{GeV}, 2.5 \mathrm{ab}^{-1}$ at $1.5 \mathrm{TeV}$, and $5 \mathrm{ab}^{-1}$ at $3 \mathrm{TeV}$ expected to be collected in the Compact Linear Collider (CLIC) project [63-66].

In our model, the triple Higgs coupling $\lambda_{h h h}$ gets modified due to the mixing with the scalar $\eta$, and in addition, it receives new one-loop contributions by the scalar $\eta$ and the new gauge bosons $A_{i}$. Taking these contributions into account, we can parametrize $\lambda_{h h h}$ as

$$
\lambda_{h h h}=\lambda_{h h h}^{\mathrm{SM}}\left(1+\Delta_{h h h}\right),
$$

where the one-loop triple Higgs coupling in the SM is given by [67]

$$
\lambda_{h h h}^{\mathrm{SM}} \simeq \frac{3 m_{h}^{2}}{v}\left[1-\frac{m_{t}^{4}}{\pi^{2} v^{2} m_{h}^{2}}\right] .
$$

According to the ILC physics group, the triple Higgs coupling can be measured at $\sqrt{s}=500 \mathrm{GeV}$ within the integrated luminosity $\mathcal{L}=500 \mathrm{fb}^{-1}$ with an accuracy less than or equal to $20 \%$ [62]. This implies that for the parameters space where $m_{\eta} \gg m_{h},{ }^{3}$ our model can be tested at future linear colliders. Here, we estimate the parameter $\Delta_{h h h}$ following [68], where the Higgs triple self-coupling can be considered as the third derivative of the Higgs oneloop effective potential

$$
\lambda_{h h h}=\frac{\partial^{3} V_{\text {eff }}}{\partial h^{3}},
$$

where the zero-temperature one-loop effective potential $V_{\text {eff }}\left(h^{\prime}, \eta^{\prime}\right)$ is described in Appendix A. Therefore, one writes

$$
\lambda_{h h h}=\left.\left\{c_{\beta}^{3} \frac{\partial^{3} V_{\text {eff }}}{\partial h^{\prime 3}}-3 c_{\beta}^{2} s_{\beta} \frac{\partial^{3} V_{\text {eff }}}{\partial \eta^{\prime} \partial h^{\prime 2}}+3 c_{\beta} s_{\beta}^{2} \frac{\partial^{3} V_{\text {eff }}}{\partial \eta^{\prime 2} \partial h^{\prime}}-s_{\beta}^{3} \frac{\partial^{3} V_{\text {eff }}}{\partial \eta^{\prime 3}}\right\}\right|_{h^{\prime}=\eta^{\prime}=0} .
$$

\footnotetext{
${ }^{3}$ In this model, the relevant process that probes the triple Higgs coupling at the ILC $\left(e^{+} e^{-} \rightarrow Z h h @ 500 \mathrm{GeV}\right)$ occurs via another Feynman diagram mediated by the new scalar $\eta$. Therefore, if $m_{\eta} \gtrsim 400 \mathrm{GeV} \gg m_{h}$ this new diagram is subleading and therefore the triple Higgs coupling can be probed similarly to the case of single Higgs models.
}

At tree level, these scalar triple couplings correspond to the $\rho_{h}$ parameter given in (15). For the benchmark points used previously, we show the Higgs triple coupling relative enhancement in Fig. 9.

The effect of these extra contributions can be either constructive or destructive according to the $\eta$ mass range. For instance, from Fig. 11, one notices that for scalar mass 


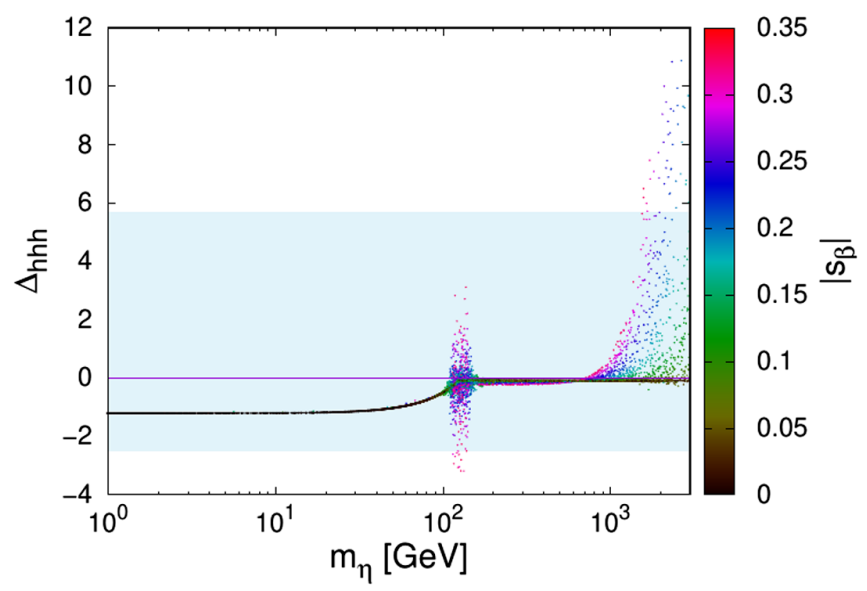

FIG. 9. The triple Higgs relative enhancement $\Delta_{h h h}$ versus the new scalar mass for the previously used benchmarks points, where the palette shows the scalar mixing. The sky-blue band represents the allowed values of $\Delta_{h h h}$ by the recent ATLAS measurements [40].

$m_{\eta}<m_{h}$ the coupling (44) is almost suppressed, while for the degenerate case $m_{\eta} \sim m_{h}$, we could have either enhancement or suppression. For $\eta$ mass larger than $1.2 \mathrm{TeV}$, some benchmark points are already suppressed by the ATLAS recent measurement [40].

\section{B. The di-Higgs production}

The di-Higgs production is not only interesting as its measurement allows one to determine the triple Higgs couplings but also to describe the Electroweak Symmetry Breaking (EWSB), i.e., it occurs via one Higgs or more. The triple Higgs coupling can be measured directly in di-Higgs boson production at ILC through double Higgs-strahlung of $W$ or $Z$ bosons [69-73], $W W$ or $Z Z$ fusion [71-77], also through gluon-gluon fusion in $p p$ collisions at the LHC [78-80]. The Higgs pair production processes in this model can be achieved via three Feynman diagrams as shown in Fig. 10. Indeed, there are two triangle diagrams mediated by the Higgs field $h$ and the new singlet scalar field $\eta$ instead of one diagram in the SM.

In the SM, the di-Higgs production cross section has three contributions:

$$
\sigma^{\mathrm{SM}}(h h)=\sigma_{\square}+\sigma_{\triangle}+\sigma_{\triangle \square},
$$

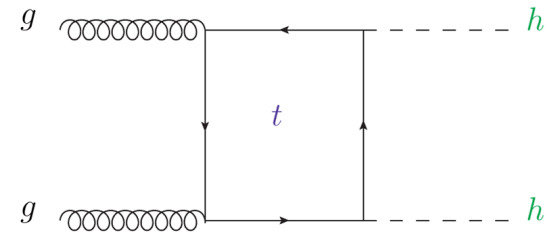

(a) which correspond to the box $\left(\sigma_{\square}=70.1 \mathrm{fb}\right)$, triangle $\left(\sigma_{\triangle}=9.66 \mathrm{fb}\right)$, and interference $\left(\sigma_{\triangle \square}=-49.9 \mathrm{fb}\right)$, respectively [81]. In this model, the di-Higgs production cross section can be written as

$$
\sigma(h h)=\xi_{1} \sigma_{\square}+\xi_{2} \sigma_{\triangle}+\xi_{3} \sigma_{\triangle \square},
$$

where the SM corresponds to $s_{\beta}=0$, i.e., $\xi_{1}=\xi_{2}=$ $\xi_{3}=1$. The coefficients $\xi_{i}$ in our model are modified with respect to the SM as

$$
\begin{aligned}
& \xi_{1}=c_{\beta}^{4}, \quad \xi_{2}=\left(c_{\beta} \frac{\rho_{h}}{\lambda_{h h h}^{S M}}+s_{\beta} \frac{\rho_{2}}{\lambda_{h h h}^{\mathrm{SM}}} \frac{s-m_{h}^{2}}{s-m_{\eta}^{2}}\right)^{2}, \\
& \xi_{3}=c_{\beta}^{2}\left(c_{\beta} \frac{\rho_{h}}{\lambda_{h h h}^{\mathrm{SM}}}+s_{\beta} \frac{\rho_{2}}{\lambda_{h h h}^{\mathrm{SM}}} \frac{s-m_{h}^{2}}{s-m_{\eta}^{2}}\right),
\end{aligned}
$$

with $\rho_{h}$ and $\rho_{2}$ are defined in (15), $\lambda_{h h h}^{\mathrm{SM}}$ the Higgs triple coupling in the SM is given in (43), and $\sqrt{s}$ is the CM collision energy, which we will consider to be $\sqrt{s}=14 \mathrm{TeV}$. In Fig. 11, left, we show the di-Higgs production cross section (47) at LHC14 scaled by its SM value versus the new scalar mass $m_{\eta}$ and the scalar mixing (in the palette), and in Fig. 11, right, we present the parameters $\xi$ 's (48) for the benchmark points used previously.

Clearly for lighter new scalar $m_{\eta}<m_{h}$ the di-Higgs cross section is larger than the SM values by around $180 \%$, while, for heavier $\eta$ scalar $m_{\eta}>m_{h}$, it lies between -92 and $3000 \%$. Indeed, not all of the benchmark points would be in agreement with current data. In order to understand these results, Fig. 11, right, shows the relation between the parameters $\xi_{2}$ and $\xi_{3}$, where $\xi_{1}$ is shown in the palette. Since the interference term in (47) is negative, then the benchmark points above the straight line $\xi_{2}=\xi_{3}$ in Fig. 11, right, correspond to larger cross-section values. For lighter $\eta$ scalar $m_{\eta}<m_{h} / 2$, where the undetermined Higgs decay channel $h \rightarrow \eta \eta$ is open, more negative searches can be used to constrain the parameters space, especially via the signatures $h \rightarrow \eta \eta \rightarrow b b \mu \mu$ [82], $h \rightarrow \eta \eta \rightarrow 4 b$ [83], and $h \rightarrow \eta \eta \rightarrow \gamma \gamma j j$ [84]. This analysis is postponed for a future work [85].

In order to determine which of the constraints is responsible for shaping the parameters space we reproduce a plot with the parameters as shown in Fig. 4, top left,

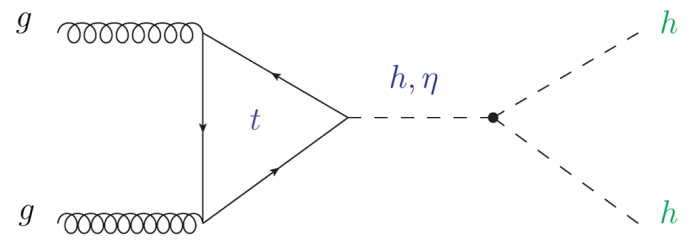

(b)

FIG. 10. Feynman diagrams that contribute to the di-Higgs production via gluon fusion. The left (right) diagram is referred to be the box (triangle) in the literature. 


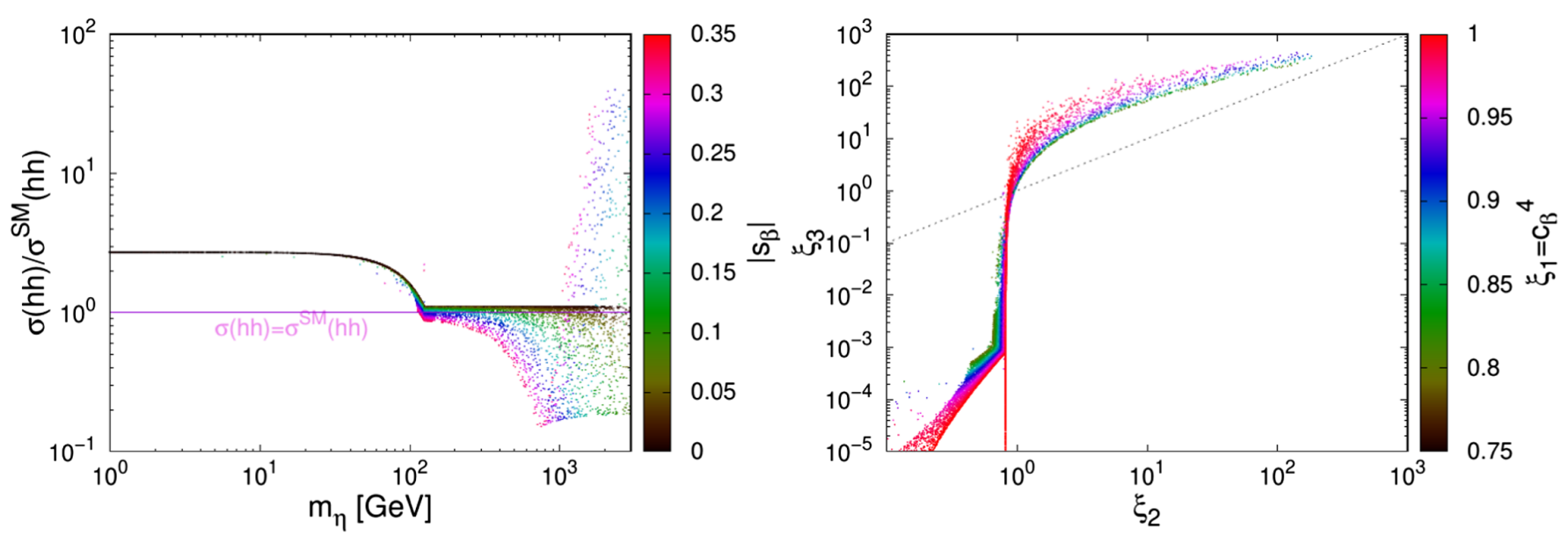

FIG. 11. Left: the di-Higgs production cross section at $14 \mathrm{TeV}$, scaled by the SM value, where the palette shows the scalar mixing. Right: the parameters $\xi$ 's (48) for the benchmark points used previously. The dashed line represents $\xi_{2}=\xi_{3}$, above which the destructive interference contribution in (47) is larger, and hence the total cross section gets reduced.

without the requirements of the observed value of the relic density and/or the DD bounds, as done in [86]. This would show also the impact on the collider observables presented in Fig. 9 and Fig. 11. The impact of the DM requirements on the parameters space and on the collider observables is shown in Fig. 12.
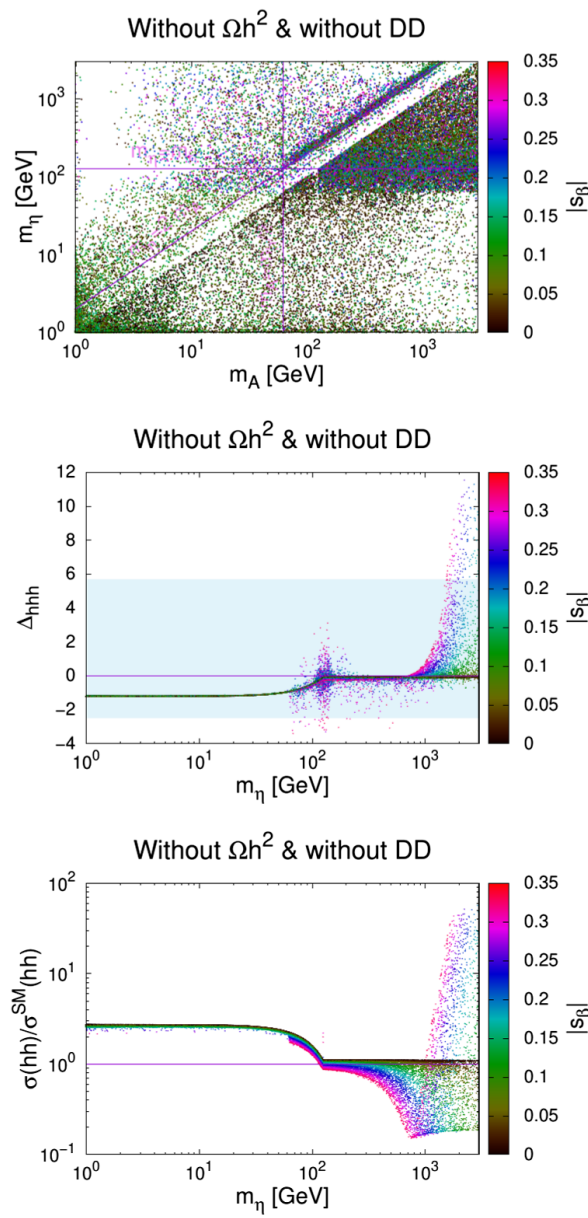
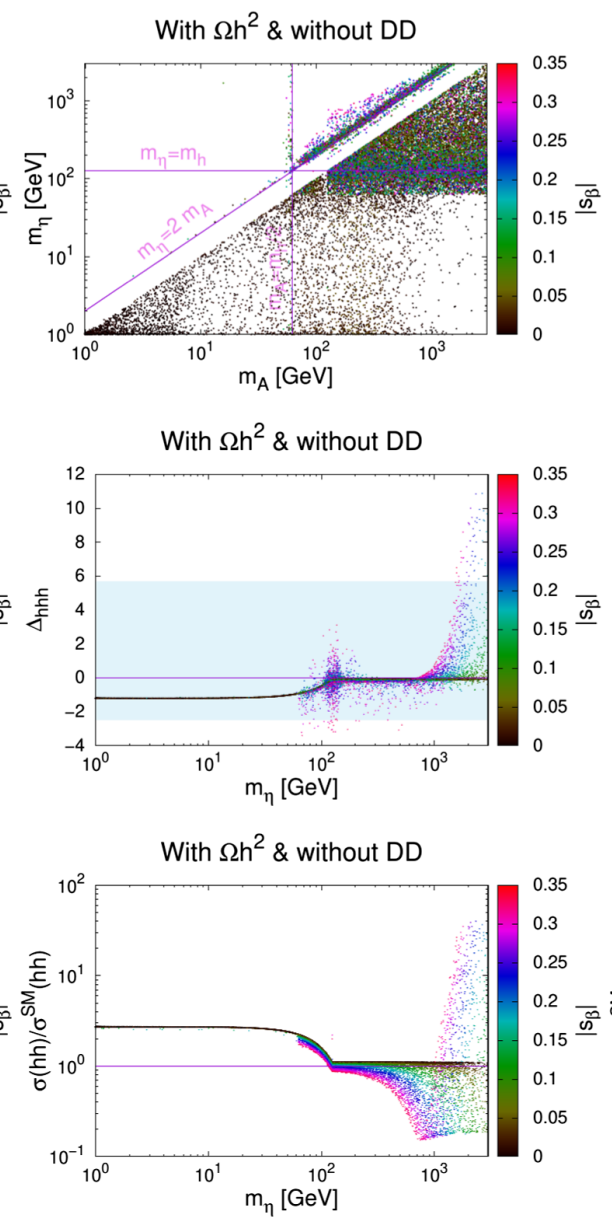
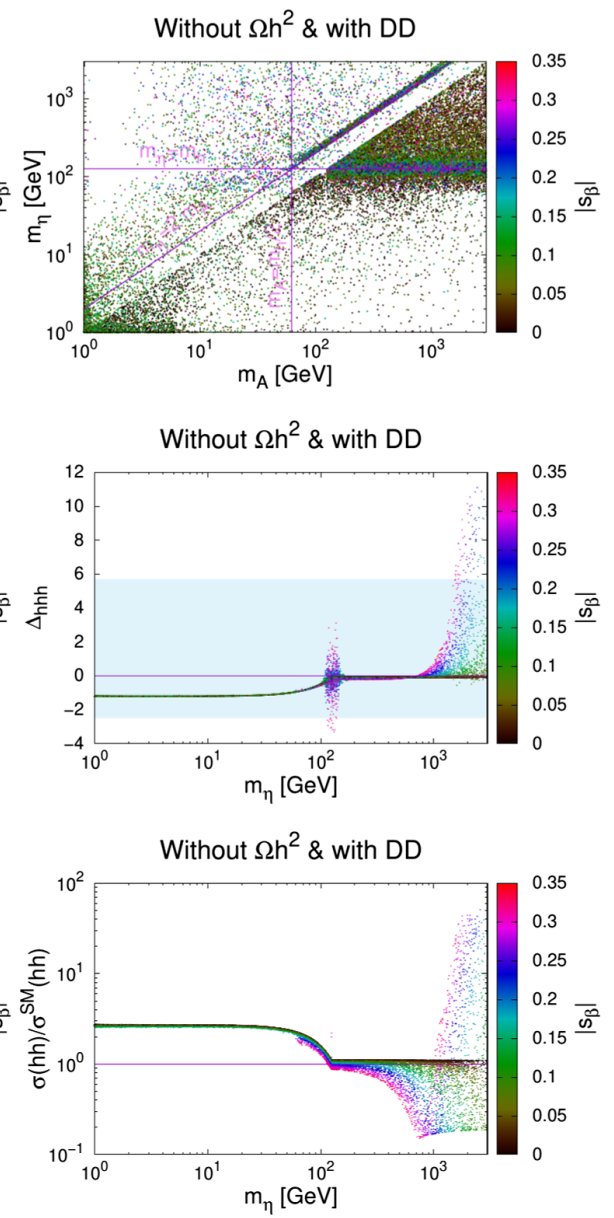

FIG. 12. The parameters space shown in Fig. 4, top left (top), the triple Higgs coupling relative enhancement (middle), and the di-Higgs production at LHC@14 (bottom), reproduced without the constraints of both the relic density and the DD (left), without the DD cross-section bound (middle), and without the relic density (right). 
Clearly, the constraint coming from the relic density is the most severe one since after imposing it most of the parameter space is excluded, especially the regions above $m_{\eta}=2 m_{A}$ and in between $m_{\eta}=2 m_{A}$ and $m_{\eta}=m_{A}$ in Fig. 4, top left. For the triple Higgs coupling relative enhancement, the impact of the relic density bound is more important than the DD one; however, for the di-Higgs production cross section, it is not clear which bound has the greater impact.

\section{CONCLUSION}

In this work, we have considered an extension of the SM by enlarging the SM gauge symmetry by a non-Abelian gauge group $\mathrm{SU}(2)_{\mathrm{HS}}$. In this case, the scalar sector of the model contains an extra new Higgs doublet that is required to spontaneously break the $\mathrm{SU}(2)_{\mathrm{HS}}$ gauge symmetry. The mixing of the extra doublet with the SM Higgs results in the mass eigenstates basis and gives two scalars denoted by $h$ and $\eta$. In our analysis, we identified $h$ as the SM neutral Higgs-like boson and adopted its mass to be $125 \mathrm{GeV}$. An important feature of the model is that the new gauge bosons, $A_{i}^{\mu}$ with $i=1,2,3$, associated with the group $\mathrm{SU}(2)_{\mathrm{HS}}$ are exactly degenerate in mass and can serve as vector DM candidates that interact with the SM through the Higgs portal.

To investigate such a possibility for DM candidate, we first considered all relevant constraints on the model, which include both theoretical and experimental ones, such as perturbative unitarity, vacuum stability, perturbativity, experimental bound on the DM direct detection, the observed DM relic density, and the constraints from the Higgs decay where the invisible or/and undetermined branching ratio where the Higgs total decay width must respect the existing experimental constraints. As a result, we showed that it is possible to have viable parameters space in which the masses of the DM candidates lie from few $\mathrm{GeV}$ to the $\mathrm{TeV}$ scale, which is still within the reach of high- energy collider experiments. Regarding the limits from direct detection DM experiments, it is easily accommodated in this model for most of the values of DM and new scalar masses. In addition, the observed relic density values can be achieved by many annihilation channels according to the DM mass, and on top of that, the coannihilation $\left(A_{i} A_{j} \rightarrow h A_{k}\right)$ effect could be important as it may reach $25 \%$ of the total thermally averaged annihilation cross section.

In our work, we considered the conditions of perturbativity, vacuum stability, and perturbative unitarity, but these conditions may not be fulfilled at higher scales. By running the quartic scalar and gauge couplings at higher scale $\Lambda$ using the RGE (25), we found that 16.5, 40, and 98.7\% of the benchmark points will be ruled out due to these conditions at scale values $\Lambda=100 \mathrm{TeV}, 10^{4} \mathrm{TeV}, m_{\text {Planck }}$, respectively.
In the decoupling limit $m_{\eta} \gg m_{h}$, as in many extensions of the SM, the Higgs mixing effects and the presence of new fields coupled to the Higgs doublet induce significant corrections to the SM prediction of the triple Higgs selfcouplings $\lambda_{h h h}=\left(1+\Delta_{h h h}\right) \lambda_{h h h}^{\mathrm{SM}}$. We have found that, up to one-loop level, the effect of the new scalar $\eta$ and the vector DM $A_{i}$ lead to a relative enhancement $\left(\Delta_{h h h}\right)$ that lies between -250 and $+1200 \%$. Indeed, part of these benchmark points are already excluded by the recent measurements by ATLAS [40]. However, in the case $m_{\eta}<m_{h}$, the cross section of the di-Higgs gets enhanced by around $180 \%$, which makes the signatures $h \rightarrow \eta \eta \rightarrow b b \mu \mu$, $h \rightarrow \eta \eta \rightarrow 4 b$, and $h \rightarrow \eta \eta \rightarrow \gamma \gamma j j$ very useful to put more constraints on the model free parameters.

\section{ACKNOWLEDGMENTS}

N. B. wants to thank the Physics department at Süleyman Demirel University (Turkey) for the warm hospitality during part of this work. The work of A. A. is supported by the University of Sharjah under the Grants No. 21021430100 "Extended Higgs Sectors at Colliders: Constraints \& Predictions" and No. 21021430107 "Hunting for New Physics at Colliders." The work of S. N. is supported by the United Arab Emirates University (UAEU) research fund, UAEU Program for Advanced Research (UPAR) Grant No. 12 S004. A. A. and S. N. acknowledge the support from the ICTP-Associate program.

\section{APPENDIX A: THE ONE-LOOP EFFECTIVE POTENTIAL}

The zero-temperature one-loop effective potential can be given in the $\overline{D R}$ scheme by

$$
\begin{aligned}
V_{\text {eff }}\left(h^{\prime}, \eta^{\prime}\right)= & V^{0}\left(h^{\prime}, \eta^{\prime}\right)+\frac{1}{64 \pi^{2}} \sum_{i} n_{i} m_{i}^{4}\left(h^{\prime}, \eta^{\prime}\right) \\
& \times\left(\log \left(\frac{m_{i}^{2}\left(h^{\prime}, \eta^{\prime}\right)}{\Lambda^{2}}\right)-\frac{3}{2}\right),
\end{aligned}
$$

where, $V^{0}\left(h^{\prime}, \eta^{\prime}\right)$ is the tree-level potential, $n_{i}$ is the number of internal degrees of freedom of the $i$ th particle $\left(n_{h}=n_{\eta}=1, n_{Z}=3, n_{W}=6, n_{t}=-12\right.$ and $\left.n_{A_{i}}=9\right)$. Here, $m_{i}^{2}\left(h^{\prime}, \eta^{\prime}\right)$ are the field-dependent squared masses, and $\Lambda$ is the renormalization scale, which we will choose to be the Higgs mass $\Lambda=125.18 \mathrm{GeV}$.

Here, we give the field-dependent squared masses $m_{i}\left(h^{\prime}, \eta^{\prime}\right)$ for all the contributing particles, so we have the field-dependent masses of the Goldstone bosons $\chi$ and $\xi$ :

$m_{\chi}^{2}=\mu^{2}+\lambda h^{\prime 2}+\frac{\lambda_{m}}{2} \eta^{\prime 2}, \quad m_{\xi}^{2}=\mu_{\phi}^{2}+\lambda_{\phi} \eta^{\prime 2}+\frac{\lambda_{m}}{2} h^{\prime 2}$.

The field-dependent masses of the electroweak gauge bosons and top quark are given in the symmetric phase (i.e., for $\left\langle h^{\prime}\right\rangle=\left\langle\phi^{\prime}\right\rangle=0$ ) by 


$$
\begin{array}{rlrl}
m_{t}^{2} & =\frac{y_{t}^{2}}{2} h^{\prime 2}, & m_{W}^{2}=\frac{g_{2}^{2}}{4} h^{2}, & m_{W^{3}}^{2}=\frac{g_{2}^{2}}{4} h^{\prime 2}, \\
m_{W^{3}-B}^{2}=\frac{g_{1}^{2}}{4} h^{\prime 2}, & m_{B}^{2}=\frac{g_{1}^{2}}{4} h^{\prime 2}, & m_{A}^{2}=\frac{g_{\phi}^{2}}{4} \eta^{\prime 2},
\end{array}
$$

where the diagonalization of the $\left\{W^{3}-B\right\}$ matrix gives $m_{\gamma}=0$ and $m_{Z}^{2}=\frac{\left(g_{1}^{2}+g_{2}^{2}\right)}{4} h^{\prime 2}$. Here, $y_{t}$ denotes the top-quark Yukawa coupling, and $g_{1}, g_{2}$, and $g_{\phi}$ are the gauge couplings of $U(1)_{Y}, \mathrm{SU}(2)_{L}$, and $\mathrm{SU}(2)_{\mathrm{HS}}$, respectively.

The field-dependent masses of $h$ and $\eta$ can be obtained as the eigenvalues of the squared mass matrix in the basis $\left\{h^{\prime}, \eta^{\prime}\right\}$, which is given by

$$
M^{2}=\left(\begin{array}{ll}
A & C \\
C & B
\end{array}\right)
$$

with $\quad A=\mu^{2}+3 \lambda h^{\prime 2}+\frac{\lambda_{m}}{2} \eta^{\prime 2}, B=\mu_{\phi}^{2}+3 \lambda_{\phi} \eta^{\prime 2}+\frac{\lambda_{m}}{2} h^{\prime 2}$, $C=\lambda_{m} h^{\prime} \eta^{\prime}$. Then, the field-dependent eigenmasses are given by $m_{h, \eta}^{2}\left(h^{\prime}, \eta^{\prime}\right)=\frac{1}{2}\left(A+B \mp \sqrt{(A-B)^{2}+C^{2}}\right)$.

\section{APPENDIX B: THE PARAMETERS IN THE CROSS SECTION FOR $h h, \eta \eta, h \eta, A h$, AND $A \eta$}

Here, we give the formulas of the parameters used in the cross section of the final states $h h, \eta \eta, h \eta, A h$, and $A \eta$ given in (34), (35), and (36). We denote by $H$ either $h$ or $\eta$, so the parameters are given by

$$
\begin{aligned}
A= & \frac{2 m_{H}^{2}-s}{2 m_{A}^{2}}, \quad B=\frac{s}{2 m_{A}^{2}} \sqrt{\left(1-\frac{4 m_{H}^{2}}{s}\right)\left(1-\frac{4 m_{A}^{2}}{s}\right)}, \quad Q_{0}=\frac{\sqrt{1-\frac{4 m_{H}^{2}}{s}}}{16 \pi}, \\
Q_{1}= & \frac{2 q^{4}}{9 v_{\phi}^{4} m_{A}^{4}}\left(48 m_{A}^{8}-32 m_{A}^{6} m_{H}^{2}+24 m_{A}^{4} m_{H}^{4}-16 m_{A}^{4} m_{H}^{2} s+4 m_{A}^{4} s^{2}-8 m_{A}^{2} m_{H}^{6}+4 m_{A}^{2} m_{H}^{4} s+m_{H}^{8}\right), \\
Q_{2}= & \frac{4 q^{4}}{9 v_{\phi}^{4} m_{A}^{2}\left(s-2 m_{H}^{2}\right)}\left(-48 m_{A}^{8}+32 m_{A}^{6} m_{H}^{2}-8 m_{A}^{4} m_{H}^{4}+4 m_{A}^{4} s^{2}-8 m_{A}^{2} m_{H}^{6}+4 m_{A}^{2} m_{H}^{2} s^{2}-2 m_{A}^{2} s^{3}\right. \\
& \left.+3 m_{H}^{8}-m_{H}^{4} s^{2}\right)+\frac{4 q^{2} \mathfrak{R}(R)}{9 v_{\phi}^{4} m_{A}^{2}\left(s-2 m_{H}^{2}\right)}\left(-48 m_{A}^{6} m_{H}^{2}+24 m_{A}^{6} s+16 m_{A}^{4} m_{H}^{4}-4 m_{A}^{4} s^{2}-4 m_{A}^{2} m_{H}^{6}\right. \\
& \left.+10 m_{A}^{2} m_{H}^{4} s-8 m_{A}^{2} m_{H}^{2} s^{2}+2 m_{A}^{2} s^{3}-2 m_{H}^{6} s+m_{H}^{4} s^{2}\right), \\
Q_{3}= & \frac{4 q^{4}}{9 v_{\phi}^{4}}\left(-4 m_{A}^{4}+4 m_{A}^{2} m_{H}^{2}-6 m_{A}^{2} s+m_{H}^{4}+2 m_{H}^{2} s\right)+\frac{8 q^{2} \mathfrak{R}(R)}{9 v_{\phi}^{4}}\left(-4 m_{A}^{2} m_{H}^{2}+4 m_{A}^{2} s-m_{H}^{2} s\right)+\frac{|R|^{2}}{9 v_{\phi}^{4}}\left(12 m_{A}^{4}-4 m_{A}^{2} s+s^{2}\right), \\
Q_{4}= & \frac{4 m_{A}^{2} q^{4}}{9 v_{\phi}^{4}\left(2 m_{H}^{2}-s\right)}\left(8 m_{A}^{4}-8 m_{A}^{2} m_{H}^{2}+8 m_{A}^{2} s+2 m_{H}^{4}-4 m_{H}^{2}+s^{2}\right)+\frac{4 m_{A}^{2} q^{2} \mathfrak{R}(R)}{9 v_{\phi}^{4}\left(2 m_{H}^{2}-s\right)}\left(4 m_{A}^{2} m_{H}^{2}-2 m_{A}^{2} s+2 m_{H}^{2} s-s^{2}\right), \\
Q_{5}= & -\frac{2 q^{4} m_{A}^{4}}{3 v_{\phi}^{4}}, \quad Q_{6}=\frac{4 q^{4} m_{A}^{6}}{9 v_{\phi}^{4}\left(2 m_{H}^{2}-s\right)},
\end{aligned}
$$

with $\{q, R\}=\left\{s_{\beta}, \frac{c_{\beta} \rho_{2} v_{\phi}}{s-m_{\eta}^{2}+i m_{\eta} \Gamma_{\eta}}-\frac{s_{\beta} \rho_{h} v_{\phi}}{s-m_{h}^{2}+i m_{h} \Gamma_{h}}+s_{\beta}^{2}\right\}$ for $h$, and $\{q, R\}=\left\{-c_{\beta}, \frac{s_{\beta} \rho_{\eta} v_{\phi}}{s-m_{\eta}^{2}+i m_{\eta} \Gamma_{\eta}}+\frac{c_{\beta} \rho_{1} v_{\phi}}{s-m_{h}^{2}+i m_{h} \Gamma_{h}}+c_{\beta}^{2}\right\}$ for $\eta$, where $\Gamma_{h}\left(\Gamma_{\eta}\right)$ is the total decay width of the Higgs (scalar $\eta$ ). Here $\mathfrak{R}(R)$ denotes the real part of $R$.

For the final state $h \eta$, the parameters are given by

$$
\begin{aligned}
A & =\frac{m_{h}^{2}+m_{\eta}^{2}-s}{2 m_{A}^{2}}, \quad B=\frac{1}{2 m_{A}^{2}} \sqrt{1-\frac{4 m_{A}^{2}}{s}} \sqrt{\left(s-m_{h}^{2}-m_{\eta}^{2}\right)^{2}-4 m_{h}^{2} m_{\eta}^{2}}, \\
Q_{0} & =\frac{\sqrt{\left(s-m_{h}^{2}-m_{\eta}^{2}\right)^{2}-4 m_{h}^{2} m_{\eta}^{2}}}{16 \pi s}, \quad R=-\frac{s_{\beta} \rho_{2} v_{\phi}}{s-m_{h}^{2}+i m_{h} \Gamma_{h}}+\frac{c_{\beta} \rho_{1} v_{\phi}}{s-m_{\eta}^{2}+i m_{\eta} \Gamma_{\eta}}-c_{\beta} s_{\beta},
\end{aligned}
$$




$$
\begin{aligned}
Q_{1}= & \frac{s_{2 \beta}^{2}}{36 m_{A}^{4} v_{\phi}^{4}}\left(48 m_{A}^{8}-16 m_{A}^{6} m_{\eta}^{2}-16 m_{A}^{6} m_{h}^{2}+8 m_{A}^{4} m_{\eta}^{4}+8 m_{A}^{4} m_{\eta}^{2} m_{h}^{2}-8 m_{A}^{4} m_{\eta}^{2} s+8 m_{A}^{4} m_{h}^{4}-8 m_{A}^{4} m_{h}^{2} s\right. \\
& \left.+4 m_{A}^{4} s^{2}-4 m_{A}^{2} m_{\eta}^{4} m_{h}^{2}-4 m_{A}^{2} m_{\eta}^{2} m_{h}^{4}+4 m_{A}^{2} m_{\eta}^{2} m_{h}^{2} s+m_{\eta}^{4} m_{h}^{4}\right), \\
Q_{2}= & \frac{s_{2 \beta}^{2}}{18 m_{A}^{2} v_{\phi}^{4}}\left(-4 m_{A}^{4} m_{\eta}^{2}-4 m_{A}^{4} m_{h}^{2}+8 m_{A}^{4} s+8 m_{A}^{2} m_{\eta}^{2} m_{h}^{2}-2 m_{A}^{2} m_{\eta}^{2} s-2 m_{A}^{2} m_{h}^{2} s-m_{\eta}^{4} m_{h}^{2}-m_{\eta}^{2} m_{h}^{4}\right) \\
& +\frac{\Re(R) s_{2 \beta}}{9 m_{A}^{2} v_{\phi}^{4}}\left(-24 m_{A}^{6}+4 m_{A}^{4} m_{\eta}^{2}+4 m_{A}^{4} m_{h}^{2}+4 m_{A}^{4} s-2 m_{A}^{2} m_{\eta}^{4}+2 m_{A}^{2} m_{\eta}^{2} m_{h}^{2}+2 m_{A}^{2} m_{\eta}^{2} s-2 m_{A}^{2} m_{h}^{4}\right. \\
& \left.+2 m_{A}^{2} m_{h}^{2} s-2 m_{A}^{2} s^{2}-m_{\eta}^{2} m_{h}^{2} s\right), \\
Q_{3}= & \frac{|R|^{2}}{9 v_{\phi}^{4}}\left(12 m_{A}^{4}-4 m_{A}^{2} s+s^{2}\right)+\frac{s_{2 \beta} \mathfrak{R}(R)}{9 v_{\phi}^{4}}\left(2 m_{A}^{2} m_{\eta}^{2}+2 m_{A}^{2} m_{h}^{2}-4 m_{A}^{2} s+m_{\eta}^{2} s+m_{h}^{2} s\right) \\
& +\frac{s_{2 \beta}^{2}}{36 v_{\phi}^{4}}\left(8 m_{A}^{4}-4 m_{A}^{2} m_{\eta}^{2}-4 m_{A}^{2} m_{h}^{2}+4 m_{A}^{2} s+4 m_{\eta}^{2} m_{h}^{2}+m_{\eta}^{4}+m_{h}^{4}\right), \\
Q_{4}= & -\frac{s_{2 \beta} \mathfrak{R}(R) m_{A}^{2}}{9 v_{\phi}^{4}}\left(2 m_{A}^{2}+s\right)-\frac{s_{2 \beta}^{2} m_{A}^{2}}{18 v_{\phi}^{4}}\left(m_{\eta}^{2}+m_{h}^{2}\right), \quad Q_{5}=\frac{s_{2 \beta}^{2}}{36} \frac{m_{A}^{4}}{v_{\phi}^{4}}, \quad Q_{6}=0 .
\end{aligned}
$$

For the final states $A h$ and $A \eta$, the parameters are given by

$$
\begin{aligned}
& A=\frac{m_{A}^{2}+m_{H}^{2}-s}{2 m_{A}^{2}}, \quad B=\frac{1}{2 m_{A}^{2}} \sqrt{1-\frac{4 m_{A}^{2}}{s}} \sqrt{\left(s-m_{H}^{2}-m_{A}^{2}\right)^{2}-4 m_{H}^{2} m_{A}^{2}}, \\
& Q_{0}=\frac{\sqrt{\left(s-m_{H}^{2}-m_{A}^{2}\right)^{2}-4 m_{H}^{2} m_{A}^{2}}}{16 \pi s} \text {, } \\
& Q_{1}=-\frac{q^{2}}{36 m_{A}^{4} v_{\phi}^{4}}\left(20 m_{A}^{8}+588 m_{A}^{6} m_{H}^{2}+256 m_{A}^{6} s-461 m_{A}^{4} m_{H}^{4}-352 m_{A}^{4} m_{H}^{2} s-96 m_{A}^{4} s^{2}\right. \\
& \left.+72 m_{A}^{2} m_{H}^{6}+108 m_{A}^{2} m_{H}^{4} s+128 m_{A}^{2} m_{H}^{2} s^{2}-36 m_{H}^{4} s^{2}\right), \\
& Q_{2}=-\frac{q^{2}}{18 m_{A}^{2} v_{\phi}^{4}\left(s-m_{A}^{2}\right)}\left(336 m_{A}^{8}-165 m_{A}^{6} m_{H}^{2}-264 m_{A}^{6} s-37 m_{A}^{4} m_{H}^{4}+241 m_{A}^{4} m_{H}^{2} s\right. \\
& \left.+128 m_{A}^{4} s^{2}-21 m_{A}^{2} m_{H}^{4} s-88 m_{A}^{2} m_{H}^{2} s^{2}-32 m_{A}^{2} s^{3}-18 m_{H}^{4} s^{2}+36 m_{H}^{2} s^{3}\right), \\
& Q_{3}=\frac{q^{2}}{36 v_{\phi}^{4}\left(s-m_{A}^{2}\right)^{2}}\left(-591 m_{A}^{8}+480 m_{A}^{6} m_{H}^{2}-302 m_{A}^{6} s+m_{A}^{4} m_{H}^{4}-416 m_{A}^{4} m_{H}^{2} s\right. \\
& \left.+189 m_{A}^{4} s^{2}+6 m_{A}^{2} m_{H}^{4} s+104 m_{A}^{2} m_{H}^{2} s^{2}-100 m_{A}^{2} s^{3}+9 m_{H}^{4} s^{2}-72 m_{H}^{2} s^{3}+36 s^{4}\right), \\
& Q_{4}=\frac{q^{2} m_{A}^{2}}{18 v_{\phi}^{4}\left(s-m_{A}^{2}\right)^{2}}\left(-153 m_{A}^{6}-69 m_{A}^{4} m_{H}^{2}+164 m_{A}^{4} s-2 m_{A}^{2} m_{H}^{2} s-29 m_{A}^{2} s^{2}-9 m_{H}^{2} s^{2}+18 s^{3}\right) \text {, } \\
& Q_{5}=\frac{q^{2} m_{A}^{4}}{36 v_{\phi}^{4}\left(s-m_{A}^{2}\right)^{2}}\left(153 m_{A}^{4}-2 m_{A}^{2} s+9 s^{2}\right), \quad Q_{6}=0,
\end{aligned}
$$

with $\left\{m_{H}, q^{2}\right\}=\left\{m_{h}, s_{\beta}\right\},\left\{m_{\eta},-c_{\beta}\right\}$ for the final states $A h$ and $A \eta$, respectively. 
[1] F. Zwicky, Helv. Phys. Acta 6, 110 (1933); Gen. Relativ. Gravit. 41, 207 (2009); E. Corbelli and P. Salucci, Mon. Not. R. Astron. Soc. 311, 441 (2000); D. P. Roy, arXiv: physics/0007025.

[2] N. Aghanim et al. (Planck Collaboration), Astron. Astrophys. 641, A6 (2020).

[3] For example: N. G. Deshpande and E. Ma, Phys. Rev. D 18, 2574 (1978); S. Hannestad, A. Mirizzi, G. G. Raffelt, and Y. Y. Y. Wong, J. Cosmol. Astropart. Phys. 04 (2008) 019; T. Hambye and M. H. G. Tytgat, Phys. Lett. B 659, 651 (2008); A. Ahriche and S. Nasri, Phys. Rev. D 85, 093007 (2012); C. Gross, O. Lebedev, and T. Toma, Phys. Rev. Lett. 119, 191801 (2017); Y. Abe, T. Toma, and K. Tsumura, J. High Energy Phys. 05 (2020) 057; N. Okada, D. Raut, and Q. Shafi, arXiv:2001.05910; A. Ahmed, S. Najjari, and C. B. Verhaaren, J. High Energy Phys. 06 (2020) 007; J. McDonald, Phys. Rev. D 50, 3637 (1994); J. McDonald, Phys. Rev. Lett. 88, 091304 (2002); C. P. Burgess, M. Pospelov, and T. ter Veldhuis, Nucl. Phys. B619, 709 (2001); C. Boehm and P. Fayet, Nucl. Phys. B683, 219 (2004); C. Boehm, Y. Farzan, T. Hambye, S. PalomaresRuiz, and S. Pascoli, Phys. Rev. D 77, 043516 (2008); V. Barger, P. Langacker, M. McCaskey, M. J. Ramsey-Musolf, and G. Shaughnessy, Phys. Rev. D 77, 035005 (2008); S. Andreas, T. Hambye, and M. H. G. Tytgat, J. Cosmol. Astropart. Phys. 10 (2008) 034.

[4] For example: P. Nath and R. L. Arnowitt, arXiv:hep-ph/ 9610460; N. Baouche and A. Ahriche, Phys. Rev. D 96, 055029 (2017); L. Covi, L. Roszkowski, R. Ruiz de Austri, and M. Small, J. High Energy Phys. 06 (2004) 003; M. Pospelov, A. Ritz, and M. B. Voloshin, Phys. Lett. B 662, 53 (2008); G. Belanger, A. Pukhov, and G. Servant, J. Cosmol. Astropart. Phys. 01 (2008) 009; L. Covi, M. Grefe, A. Ibarra, and D. Tran, J. Cosmol. Astropart. Phys. 01(2009) 029; S. Ipek, D. McKeen, and A. E. Nelson, Phys. Rev. D 90, 055021 (2014); K. Ghorbani, J. Cosmol. Astropart. Phys. 01 (2015) 015; A. Ahriche and S. Nasri, J. Cosmol. Astropart. Phys. 07 (2013) 035; A. Ahriche, C. S. Chen, K. L. McDonald, and S. Nasri, Phys. Rev. D 90, 015024 (2014); A. Ahriche, K. L. McDonald, and S. Nasri, J. High Energy Phys. 10 (2014) 167; A. Ahriche, K. L. McDonald, S. Nasri, and T. Toma, Phys. Lett. B 746, 430 (2015); A. Ahriche, K. L. McDonald, and S. Nasri, J. High Energy Phys. 02 (2016) 038; A. Ahriche, K. L. McDonald, S. Nasri, and I. Picek, Phys. Lett. B 757, 399 (2016); A. Ahriche, A. Manning, K. L. McDonald, and S. Nasri, Phys. Rev. D 94, 053005 (2016); A. Ahriche, A. Jueid, and S. Nasri, Phys. Rev. D 97, 095012 (2018); A. Ahriche, A. Arhrib, A. Jueid, S. Nasri, and A. de La Puente, Phys. Rev. D 101, 035038 (2020); A. Ahriche, A. Jueid, and S. Nasri, Phys. Lett. B 814, 136077 (2021); A. Jueid, S. Nasri, and R. Soualah, J. High Energy Phys. 04 (2021) 012; S. Baek, P. Ko, and J. Li, Phys. Rev. D 95, 075011 (2017); G. Faisel, S. Y. Ho, and J. Tandean, Phys. Lett. B 738, 380 (2014).

[5] For example: H. C. Cheng, J. L. Feng, and K. T. Matchev, Phys. Rev. Lett. 89, 211301 (2002); D. Hooper and G. D. Kribs, Phys. Rev. D 70, 115004 (2004); H. C. Cheng and I. Low, J. High Energy Phys. 09 (2003) 051; A. Birkedal, A. Noble, M. Perelstein, and A. Spray, Phys. Rev. D 74, 035002 (2006); S. Kanemura, S. Matsumoto, T. Nabeshima, and N. Okada, Phys. Rev. D 82, 055026 (2010); O. Lebedev, H. M. Lee, and Y. Mambrini, Phys. Lett. B 707, 570 (2012); T. Abe, M. Kakizaki, S. Matsumoto, and O. Seto, Phys. Lett. B 713, 211 (2012); Y. Farzan and A. R. Akbarieh, J. Cosmol. Astropart. Phys. 10 (2012) 026; J. M. Hyde, A. J. Long, and T. Vachaspati, Phys. Rev. D 89, 065031 (2014); P. Ko, W. I. Park, and Y. Tang, J. Cosmol. Astropart. Phys. 09 (2014) 013; S. Baek, P. Ko, W. I. Park, and E. Senaha, J. High Energy Phys. 05 (2013) 036; S. Baek, P. Ko, and W. I. Park, Phys. Rev. D 90, 055014 (2014); J. H. Yu, Phys. Rev. D 90, 095010 (2014); C. R. Chen, Y. K. Chu, and H. C. Tsai, Phys. Lett. B 741, 205 (2015); H. Zhang, C. S. Li, Q. H. Cao, and Z. Li, Phys. Rev. D 82, 075003 (2010); J. L. Diaz-Cruz and E. Ma, Phys. Lett. B 695, 264 (2011); S. Bhattacharya, J. L. Diaz-Cruz, E. Ma, and D. Wegman, Phys. Rev. D 85, 055008 (2012); T. Hambye and A. Strumia, Phys. Rev. D 88, 055022 (2013); H. Davoudiasl and I. M. Lewis, Phys. Rev. D 89, 055026 (2014); S. Baek, P. Ko, and W. I. Park, J. Cosmol. Astropart. Phys. 10 (2014) 067; V. V. Khoze and G. Ro, J. High Energy Phys. 10 (2014) 061; S. Fraser, E. Ma, and M. Zakeri, Int. J. Mod. Phys. A 30, 1550018 (2015); A. Karam and K. Tamvakis, Phys. Rev. D 92, 075010 (2015); T. Abe, M. Fujiwara, J. Hisano, and K. Matsushita, J. High Energy Phys. 07 (2020) 136; N. Bernal, X. Chu, C. Garcia-Cely, T. Hambye, and B. Zaldivar, J. Cosmol. Astropart. Phys. 03 (2016) 018. [arXiv: 1510.08063; V. V. Khoze and A. D. Plascencia, J. High Energy Phys. 11 (2016) 025; B. D. Sáez, F. Rojas-Abatte, and A. R. Zerwekh, Phys. Rev. D 99, 075026 (2019); C. W. Chiang, T. Nomura, and J. Tandean, J. High Energy Phys. 01 (2014) 183; C. F. Chang, X. G. He, and J. Tandean, J. High Energy Phys. 04 (2017) 107; J. Kopp, J. Liu, T. R. Slatyer, X. P. Wang, and W. Xue, J. High Energy Phys. 12 (2016) 033.

[6] T. Hambye, J. High Energy Phys. 01 (2009) 028.

[7] C. D. Carone and R. Ramos, Phys. Rev. D 88, 055020 (2013); R. Ramos, Van Que Tran, and T. C. Yuan, Phys. Rev. D 103, 075021 (2021); Z. Hu, C. Cai, Y. L. Tang, Z. H. Yu, and H. H. Zhang, J. High Energy Phys. 07 (2021) 089.

[8] Z. Hu, C. Cai, Y.L. Tang, Z.H. Yu, and H. H. Zhang, J. High Energy Phys. 07 (2021) 089; C. Cai and H. H. Zhang, arXiv:2107.13475.

[9] C. Arina, T. Hambye, A. Ibarra, and C. Weniger, J. Cosmol. Astropart. Phys. 03 (2010) 024.

[10] G. Arcadi, A. Djouadi, and M. Raidal, Phys. Rep. 842, 1 (2020).

[11] J. M. Cornwall, D. N. Levin, and G. Tiktopoulos, Phys. Rev. D 10, 1145 (1974); 11, 972(E) (1975); B. W. Lee, C. Quigg, and H. B. Thacker, Phys. Rev. D 16, 1519 (1977); B. W. Lee, C. Quigg, and H. B. Thacker, Phys. Rev. Lett. 38, 883 (1977); S. Kanemura, T. Kubota, and E. Takasugi, Phys. Lett. B 313, 155 (1993); A. G. Akeroyd, A. Arhrib, and E. M. Naimi, Phys. Lett. B 490, 119 (2000); J. Horejsi and M. Kladiva, Eur. Phys. J. C 46, 81 (2006).

[12] A. Arhrib, arXiv:hep-ph/0012353.

[13] G. Aad et al. (ATLAS Collaboration), Phys. Rev. D 101, 012002 (2020).

[14] M. Aaboud et al. (ATLAS Collaboration), Phys. Rev. Lett. 122, 231801 (2019). 
[15] ATLAS Collaboration, Combination of searches for invisible Higgs boson decays with the ATLAS experiment, Report No. ATLAS-CONF-2020-052.

[16] A. M. Sirunyan et al. (CMS Collaboration), Phys. Lett. B 793, 520 (2019).

[17] V. Khachatryan et al. (CMS Collaboration), Phys. Rev. D 92, 072010 (2015).

[18] M. Aaboud et al. (ATLAS Collaboration), Phys. Lett. B 786, 223 (2018).

[19] E. Aprile et al. (XENON Collaboration), J. Cosmol. Astropart. Phys. 04 (2016) 027.

[20] A. Falkowski, C. Gross, and O. Lebedev, J. High Energy Phys. 05 (2015) 057.

[21] M. Srednicki, R. Watkins, and K. A. Olive, Nucl. Phys. B310, 693 (1988); E. W. Kolb and M. S. Turner, Front. Phys. 69, 1 (1990), https://inspirehep.net/literature/299778.

[22] J. Hisano, S. Matsumoto, M. M. Nojiri, and O. Saito, Phys. Rev. D 71, 063528 (2005); M. Cirelli, A. Strumia, and M. Tamburini, Nucl. Phys. B787, 152 (2007); M. Cirelli and A. Strumia, Proc. Sci., IDM2008 (2008) 089 [arXiv: 0808.3867].

[23] M. Cirelli, M. Kadastik, M. Raidal, and A. Strumia, Nucl. Phys. B813, 1 (2009).

[24] O. Adriani et al. (PAMELA Collaboration), Nature (London) 458, 607 (2009).

[25] M. Ackermann et al. (Fermi-LAT Collaboration), Phys. Rev. Lett. 108, 011103 (2012).

[26] G. Aad et al. (ATLAS and CMS Collaborations), J. High Energy Phys. 08 (2016) 045.

[27] A. M. Sirunyan et al. (CMS Collaboration), Phys. Rev. Lett. 121, 121801 (2018).

[28] M. Aaboud et al. (ATLAS Collaboration), Phys. Lett. B 786, 59 (2018).

[29] A. M. Sirunyan et al. (CMS Collaboration), Eur. Phys. J. C 79, 421 (2019).

[30] M. Cepeda, S. Gori, P. Ilten, M. Kado, F. Riva, R. Abdul Khalek, A. Aboubrahim, J. Alimena, S. Alioli, A. Alves et al., CERN Yellow Rep. Monogr. 7, 221 (2019).

[31] G. Arcadi, A. Djouadi, and M. Kado, Phys. Lett. B 805, 135427 (2020).

[32] J. Billard, L. Strigari, and E. Figueroa-Feliciano, Phys. Rev. D 89, 023524 (2014).

[33] M. J. Dolan, C. Englert, and M. Spannowsky, J. High Energy Phys. 10 (2012) 112.

[34] A. Efrati and Y. Nir, arXiv:1401.0935.

[35] J. Baglio, A. Djouadi, R. Gröber, M. M. Mühlleitner, J. Quevillon, and M. Spira, J. High Energy Phys. 04 (2013) 151.

[36] M. McCullough, Phys. Rev. D 90, 015001 (2014); 92, 039903(E) (2015).

[37] M. Gorbahn and U. Haisch, J. High Energy Phys. 10 (2016) 094.

[38] F. Maltoni, D. Pagani, A. Shivaji, and X. Zhao, Eur. Phys. J. C 77, 887 (2017).

[39] G. D. Kribs, A. Maier, H. Rzehak, M. Spannowsky, and P. Waite, Phys. Rev. D 95, 093004 (2017).

[40] The ATLAS Collaboration, Report No. ATLAS-CONF2021-016.

[41] L. B. Chen, H. T. Li, H. S. Shao, and J. Wang, J. High Energy Phys. 03 (2020) 072.
[42] A. J. Barr, M. J. Dolan, C. Englert, and M. Spannowsky, Phys. Lett. B 728, 308 (2014).

[43] Q. Li, Q. S. Yan, and X. Zhao, Phys. Rev. D 89, 033015 (2014).

[44] Q. H. Cao, B. Yan, D. M. Zhang, and H. Zhang, Phys. Lett. B 752, 285 (2016).

[45] Q. Li, Z. Li, Q. S. Yan, and X. Zhao, Phys. Rev. D 92, 014015 (2015).

[46] Q. H. Cao, Y. Liu, and B. Yan, Phys. Rev. D 95, 073006 (2017).

[47] Q. H. Cao, G. Li, B. Yan, D. M. Zhang, and H. Zhang, Phys. Rev. D 96, 095031 (2017).

[48] H. J. He, J. Ren, and W. Yao, Phys. Rev. D 93, 015003 (2016).

[49] T. Huang, J. M. No, L. Pernié, M. Ramsey-Musolf, A. Safonov, M. Spannowsky, and P. Winslow, Phys. Rev. D 96, 035007 (2017).

[50] C. T. Lu, J. Chang, K. Cheung, and J. S. Lee, J. High Energy Phys. 08 (2015) 133.

[51] A. Papaefstathiou, L. L. Yang, and J. Zurita, Phys. Rev. D 87, 011301 (2013).

[52] J. H. Kim, K. Kong, K. T. Matchev, and M. Park, Phys. Rev. Lett. 122, 091801 (2019).

[53] G. Buchalla, M. Capozi, A. Celis, G. Heinrich, and L. Scyboz, J. High Energy Phys. 09 (2018) 057.

[54] J. H. Kim, M. Kim, K. Kong, K. T. Matchev, and M. Park, J. High Energy Phys. 09 (2019) 047.

[55] G. Li, L. X. Xu, B. Yan, and C. P. Yuan, Phys. Lett. B 800, 135070 (2020).

[56] J. Chang, K. Cheung, J. S. Lee, C. T. Lu, and J. Park, Phys. Rev. D 100, 096001 (2019).

[57] M. Abdughani, D. Wang, L. Wu, J. M. Yang, and J. Zhao, Phys. Rev. D 104, 056003 (2021).

[58] R. Contino, D. Curtin, A. Katz, M. L. Mangano, G. Panico, M. J. Ramsey-Musolf, G. Zanderighi, C. Anastasiou, W. Astill, G. Bambhaniya et al., CERN Yellow Rep. 3, 255 (2017).

[59] D. Gonçalves, T. Han, F. Kling, T. Plehn, and M. Takeuchi, Phys. Rev. D 97, 113004 (2018).

[60] K. Fujii, C. Grojean, M. E. Peskin, T. Barklow, Y. Gao, S. Kanemura, H. Kim, J. List, M. Nojiri, M. Perelstein et al., arXiv:1710.07621.

[61] J. de Blas, M. Cepeda, J. D’Hondt, R. K. Ellis, C. Grojean, B. Heinemann, F. Maltoni, A. Nisati, E. Petit, R. Rattazzi et al., J. High Energy Phys. 01 (2020) 139.

[62] K. Fujii, C. Grojean, M. E. Peskin, T. Barklow, Y. Gao, S. Kanemura, H. D. Kim, J. List, M. Nojiri, M. Perelstein et al., arXiv:1506.05992.

[63] J. Braathen and S. Kanemura, Eur. Phys. J. C 80, 227 (2020).

[64] P. Roloff, U. Schnoor, R. Simoniello, and B. Xu (CLICdp Collaboration), Eur. Phys. J. C 80, 1010 (2020).

[65] H. Abramowicz, A. Abusleme, K. Afanaciev, N. A. Tehrani, C. Balázs, Y. Benhammou, M. Benoit, B. Bilki, J. J. Blaising, M. J. Boland et al., Eur. Phys. J. C 77, 475 (2017).

[66] P. N. Burrows et al. (CLICdp and CLIC Collaborations), arXiv:1812.06018.

[67] S. Kanemura, S. Kiyoura, Y. Okada, E. Senaha, and C. P. Yuan, Phys. Lett. B 558, 157 (2003); S. Kanemura, 
Y. Okada, E. Senaha, and C. P. Yuan, Phys. Rev. D 70, 115002 (2004).

[68] A. Ahriche, A. Arhrib, and S. Nasri, J. High Energy Phys. 02 (2014) 042.

[69] G. J. Gounaris, D. Schildknecht, and F. M. Renard, Phys. Lett. 83B, 191 (1979).

[70] V. D. Barger, T. Han, and R. J. N. Phillips, Phys. Rev. D 38, 2766 (1988).

[71] V. A. Ilyin, A. E. Pukhov, Y. Kurihara, Y. Shimizu, and T. Kaneko, Phys. Rev. D 54, 6717 (1996).

[72] A. Djouadi, W. Kilian, M. Muhlleitner, and P. M. Zerwas, Eur. Phys. J. C 10, 27 (1999).

[73] J. Tian, K. Fujii, and Y. Gao, arXiv:1008.0921.

[74] F. Boudjema and E. Chopin, Z. Phys. C 73, 85 (1996).

[75] V. D. Barger and T. Han, Mod. Phys. Lett. A 05, 667 (1990).

[76] A. Dobrovolskaya and V. Novikov, Z. Phys. C 52, 427 (1991).
[77] A. Abbasabadi, W. W. Repko, D. A. Dicus, and R. Vega, Phys. Rev. D 38, 2770 (1988).

[78] E. W. N. Glover and J. J. van der Bij, Nucl. Phys. B309, 282 (1988).

[79] T. Plehn, M. Spira, and P. M. Zerwas, Nucl. Phys. B479, 46 (1996); B531, 655(E) (1998).

[80] S. Dawson, S. Dittmaier, and M. Spira, Phys. Rev. D 58, 115012 (1998).

[81] M. Spira, arXiv:hep-ph/9510347.

[82] ATLAS Collaboration, Report No. ATLAS-CONF-2021009.

[83] G. Aad et al. (ATLAS Collaboration), Phys. Rev. D 102, 112006 (2020).

[84] M. Aaboud et al. (ATLAS Collaboration), Phys. Lett. B 782, 750 (2018).

[85] A. Ahriche et al. (to be published).

[86] C. R. Chen, Y. X. Lin, V. Tran, and T. C. Yuan, Phys. Rev. D 99, 075027 (2019). 